for $\mathrm{LiNbO}_{3}$ given in Table II, along with the above estimate that $d_{113}^{m} \approx d_{333}^{m}$ for this material. We find that $K=-1.1\left(10^{-12}\right) \mathrm{m}^{3} / J$. Predicted values of $\delta_{113}^{m}$ and $d_{113}^{m}$ are given in Table II for some other pyroelectric crystals for which $d_{333}^{m}$ is known. The positive sign and enormous magnitude anticipated for $d_{113}^{m}$ in $\mathrm{BaTiO}_{3}$ are most interesting. All of these predictions await experimental verification.

\section{ACKNOWLEDGMENTS}

It is a pleasure to acknowledge the encourage ment of G. D. Boyd in the course of this work and the helpful comments of G. A. Coquin on the manuscript.
${ }^{1}$ G. D. Boyd, T. J. Bridges, M. A. Pollack, and E. H. Turner, Phys. Rev. Letters 26, 387 (1971). In Eq. (2) of this reference $d_{\text {eff }}$ should be $d_{\text {eff }}^{2}$.

${ }^{2}$ E. H. Turner (unpublished).

${ }^{3}$ R. C. Miller, S. C. Abrahams, R. L. Barns, J. L. Bernstein, W. A. Nordland, and E. H. Turner, Solid State Commun. 9 , 1463 (1971).

${ }^{4}$ R. C. Miller and W. A. Nordland, Opt. Commun. 1, 400 (1970); R. C. Miller and W. A. Nordland, Phys. Rev. B 2, 4896 (1970).

${ }^{5}$ C. G. B. Garrett, J. Quantum Electron. 4, 70 (1968).

${ }^{6}$ D. F. Nelson and E. H. Turner, J. Appl. Phys. 39, 3337 (1968).

${ }^{7}$ W. L. Faust and C. H. Henry, Phys. Rev. Letters 17, 1265 (1966).

${ }^{8}$ T. J. Bridges, G. D. Boyd, and M. A. Pollack, Proceedings of the Symposium on Submillimeter Waves, Vol. 20 of Microwave Research Institute Symposia Series (Polytechnic, Brooklyn, New York, 1971).

${ }^{9}$ D. R. Hamilton, J. K. Knipp, and J. H. B. Kuper,
Klystrons and Microwave Triodes (McGraw-Hill, New York, 1948).

${ }^{10} \mathrm{~A}$ more complete discussion of the orienting problem and a number of references can be found in Ref. 4 .

${ }^{11} \mathrm{~J}$. F. Nye, Physical Properties of Crystals (Clarendon, Oxford, England, 1957), p. 127.

${ }^{12}$ Proc. IRE 37, 1387 (1949).

${ }^{13}$ In Ref. 1 experiment showed $\frac{1}{3}\left|2 d_{231}^{m}+d_{123}^{m}\right| \approx\left|d_{123}^{m}\right|$, and it was deduced that $d_{121}^{m}=d_{123}^{m}$.

${ }^{14}$ D. A. Kleinman, Phys. Rev. 126, 1977 (1962).

${ }^{15}$ K. Nassau, H. J. Levinstein, and G. H. Loiacono, J. Phys. Chem. Solids 27, 989 (1966).

${ }^{16} \mathrm{~J}$. Jerphagnon, Phys. Rev. B 2,1091 (1970).

${ }^{17}$ It is not clear whether the $\delta_{A B}$ of Ref. 1 would have been more appropriate here. However, the difference in the $\delta$ 's is less than the sort of error expected in this calculation.

${ }^{18}$ S. H. Wemple, M. DiDomenico, Jr., and I. Camlibel, Appl. Phys. Letters 12, 209 (1968).

\title{
Dielectric Properties of Lithium Hydrazinium Sulfate ${ }^{\dagger}$
}

\author{
V. Hugo Schmidt* and John E. Drumheller \\ Department of Physics, Montana State University, Bozeman, Montana 59715 \\ and \\ Francis L. Howell \\ Department of Physics, University of North Dakota, Grand Forks, North Dakota 58201 \\ (Received 30 April 1971)
}

\begin{abstract}
The dc conductivity and ac dielectric susceptibility of normal and deuterated lithium hydrazinium sulfate have been measured over a wide temperature range at frequencies up to 9.33 $\mathrm{GHz}$. Over a very large temperature and frequency range the real and imaginary parts of the susceptibility are very large (up to $\epsilon^{\prime} \simeq \epsilon^{\prime \prime} \simeq 10^{6}$ ) and vary with frequency somewhat as $f^{-1 / 2}$. This unusual behavior is shown to result from the nearly one-dimensional protonic conductivity and its extreme sensitivity to barriers caused by local structural defects. Etching studies indicate that the crystal is not ferroelectric, implying that the apparent hysteresis loops result from saturation of the ac conduction.
\end{abstract}

\section{INTRODUCTION}

Lithium hydrazinium sulfate $\left(\mathrm{LiN}_{2} \mathrm{H}_{5} \mathrm{SO}_{4}\right)$ has been generally considered to be a member of the ferroelectric sulfate family ${ }^{1}$ because it exhibits what appear to be hysteresis loops, although no conclusive evidence for a ferroelectric phase transition has been found. At the same time, it exhibits unusually large protonic conductivity along the "ferroelectric" $c$ axis. ${ }^{1,2}$ It has been previously suggested that this crystal is not ferroelectric, and that its anomalous dielectric behavior is caused by protons in the hydrazinium chains which run along the $c$ axis, but no detailed explanation was given. ${ }^{3}$ We present herein a model which assumes partially blocked current flow along the hydrazinium ion chains and which predicts no hysteresis in the dc limit. Good qualitative agreement with our experimental results is ob- 


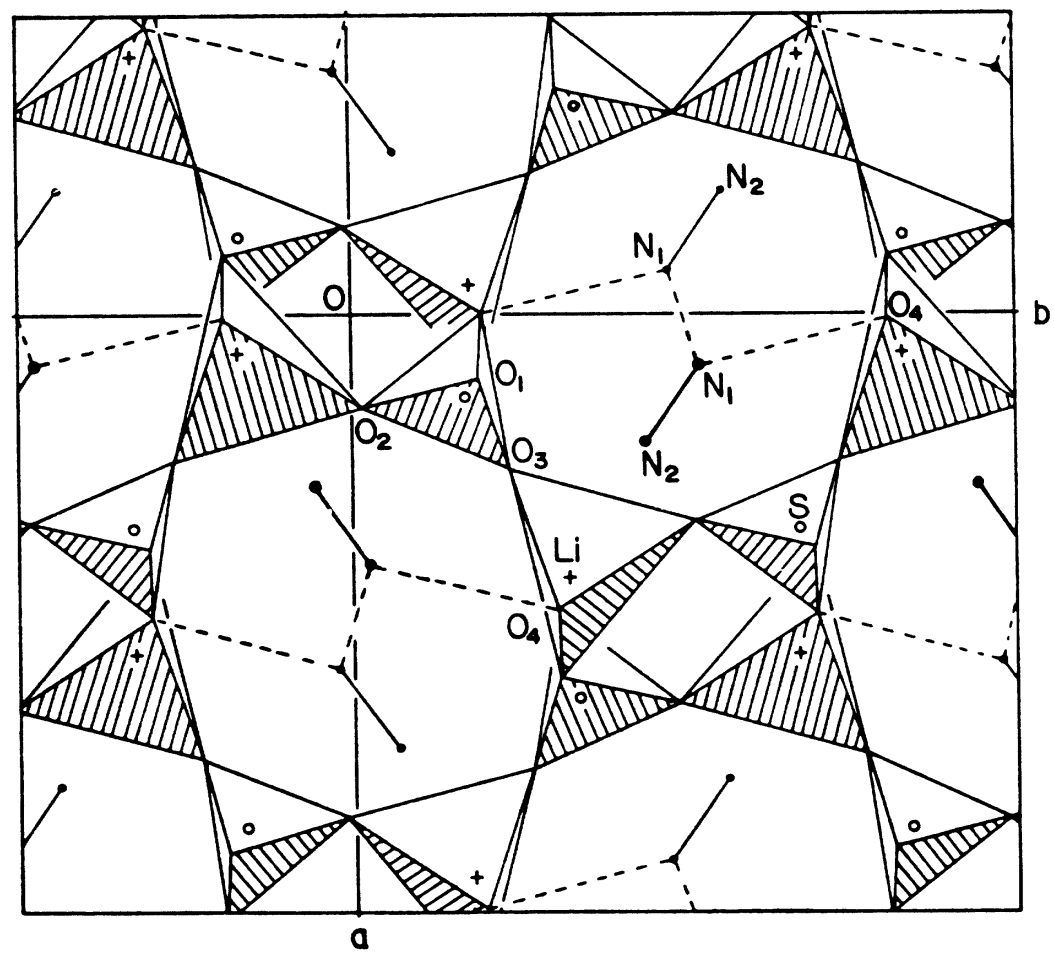

FIG. 1. Projection along $c$ axis of $\mathrm{LiN}_{2} \mathrm{H}_{5} \mathrm{SO}_{4}$ (after Brown, Ref. 4).

tained.

The dielectric behavior of $\mathrm{LiN}_{2} \mathrm{H}_{5} \mathrm{SO}_{4}(\mathrm{LiHzS})$ was first examined by Pepinsky, Vedam, Okaya, and Hoshino. ${ }^{1}$ They observed hysteresis loops from $-15{ }^{\circ} \mathrm{C}$, at which temperature the loops were asymmetric and difficult to saturate, up to $80^{\circ} \mathrm{C}$, above which the loops were difficult to interpret because of the rapid rise in electrical conductivity with temperature. They observed no dielectric peak between -196 and $140^{\circ} \mathrm{C}$, and no specific-heat anomaly between -120 and $205^{\circ} \mathrm{C}$. They concluded that the crystal is ferroelectric, with its ferroelectric axis along $c$.

Vanderkooy, Cuthbert, and Petch ${ }^{2}$ investigated the electrical conductivity of $\mathrm{LiN}_{2} \mathrm{H}_{5} \mathrm{SO}_{4}$, and found the $c$-axis dc conductivity to be given by $\left[49 \times 10^{4}\right.$ $\left.(\Omega \mathrm{cm})^{-1}\right] e^{-(0.85 \mathrm{eV} / k T)}$. At room temperature the conductivity along $c$ was over 200 times as great as along $a$ or $b$, but above $200^{\circ} \mathrm{C}$ the conductivity became virtually isotropic. A coulometer experiment with field applied along $c$ demonstrated that the current is protonic. The ac conductivity measured between 20 and $5000 \mathrm{~Hz}$ increased with increasing frequency and showed an activation energy of 0.41 $\pm 0.04 \mathrm{eV}$.

Several $\mathrm{x}$-ray structure studies have been made of $\mathrm{LiN}_{2} \mathrm{H}_{5} \mathrm{SO}_{4},{ }^{3-5}$ and neutron diffraction studies have been made for the structures of both the normal $^{6}$ and the deuterated ${ }^{7}$ material. The structure is orthorhombic, with space group $\operatorname{Pbn} 2_{1}\left(C_{2 v}^{9}\right)$. The unit cell has dimensions $a=8.969 \AA, b=9.913 \AA$, $c=5.178 \AA$, and contains four formula units. De scriptively it consists of a $\mathrm{LiSO}_{4}{ }^{-}$framework containing channels running along $c$ as shown in Fig. 1. The hydrazinium $\left(\mathrm{NH}_{2}-\mathrm{NH}_{3}{ }^{+}\right)$ions lie in these channels, with one hydrogen from each $\mathrm{NH}_{2}$ group participating in an ordered $\mathrm{N}-\mathrm{H} \cdots \mathrm{N}-\mathrm{H} \cdots$ chain which runs along $c$, as indicated in Fig. 2. The other $\mathrm{NH}_{2}$ hydrogen is bonded weakly to an oxygen. The $\mathrm{NH}_{3}{ }^{+}$nitrogens do not participate in the $\mathrm{N}-\mathrm{H} \cdots \mathrm{N}-\mathrm{H} \cdots$ chains, and their hydrogens are bonded to oxygens in the $\mathrm{LiSO}_{4}{ }^{-}$framework.

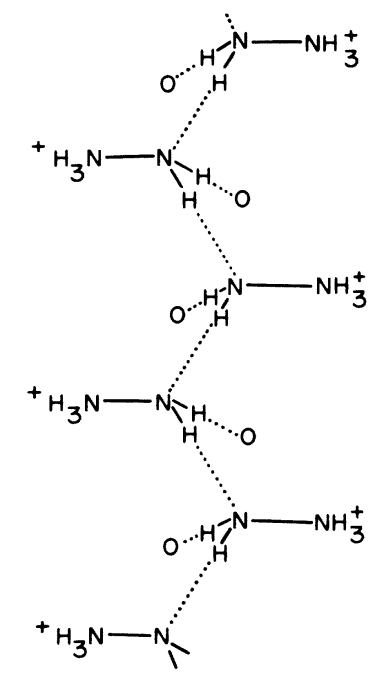

FIG. 2. Schematic arrangement of hydrazinium ions in channels running along $c$ axis (after Cuthbert and Petch, Ref. 8). 
Nuclear magnetic resonance (NMR) studies have shed some light on the possible dielectric mechanisms. The first investigation, by Cuthbert and Petch ${ }^{8}$ on the proton and $\mathrm{Li}^{7}$ spectra and second moments in $\mathrm{LiHzS}$, indicated that the $\mathrm{NH}_{2}$ and $\mathrm{NH}_{3}{ }^{+}$ groups undergo hindered rotation. They observed a rapid but continuous change in the $\mathrm{Li}^{7}$ quadrupolar splitting between 80 and $160^{\circ} \mathrm{C}$, which they interpreted as evidence for a structure change. In a subsequent study, MacClement, Pintar, and Petch ${ }^{9}$ found evidence of hydrazinium ion tumbling and diffusion at high temperature. Recently, Knispel and Petch $^{10}$ measured the proton spin-lattice relaxation time in the rotating frame $\left(T_{1 \rho}\right)$, and from the results determined the activation energies for $\mathrm{NH}_{3}{ }^{+}$ rotation, $\mathrm{NH}_{2}$ rotation, and $\mathrm{N}_{2} \mathrm{H}_{5}{ }^{+}$ion tumbling to be $0.19,0.46$, and $0.69 \mathrm{eV}$, respectively. In $\mathrm{LiN}_{2} \mathrm{D}_{5} \mathrm{SO}_{4}(\mathrm{LiDzS})$, Howell and Schmidt ${ }^{11,12}$ by means of deuteron $T_{1}$ measurements found activation energies of 0.20 and $0.50 \mathrm{eV}$ for hindered rotation of $\mathrm{ND}_{3}{ }^{+}$and $\mathrm{ND}_{2}$ groups, respectively. The $T_{1}$ measurements also indicated deuteron exchange between $\mathrm{ND}_{2}$ and $\mathrm{ND}_{3}{ }^{+}$groups, with activation energy of $0.75 \mathrm{eV}$. The quadrupolar splitting of the merged $D$ lines from the $\mathrm{ND}_{2}$ group above room temperature, when compared with the splittings of the unmerged lines below room temperature, can be explained only by assuming that the $\mathrm{ND}_{2}$ deuterons exchange positions. Knispel and Petch have shown that the $\mathrm{NH}_{2}$ group in $\mathrm{LiHzS}$ undergoes this type of hindered rotation also. Similarly the $\mathrm{NH}_{3}{ }^{+}$and $\mathrm{ND}_{3}{ }^{+}$ hindered rotations result in permutation of hydrogen locations with no net charge redistribution. Accordingly none of these hindered rotations affects the dielectric behavior.

Four explanations of the dielectric properties of $\mathrm{LiHzS}$ have been considered by others and ourselves: (a) ferroelectricity with chemically equivalent domains, (b) ferroelectricity with chemically inequivalent domains, (c) nonferroelectric partial disorder of the $\mathrm{N}-\mathrm{H} \cdots \mathrm{N}-\mathrm{H}$ dipole chains, and (d) partially blocked protonic conductivity. Niizeki and Koizumi ${ }^{3}$ were the first to cast doubt upon the ferroelectric nature of $\mathrm{LiHzS}$ by pointing out that polarization reversal for chemically equivalent domains would require considerable rotation of the $\mathrm{SO}_{4}{ }^{--}$tetrahedra, which point nearly along the positive $c$ axis. Recent experiments in our laboratory confirm their doubts. Several crystals were prepared having large $(0.2$ to $0.5 \mathrm{~cm}^{2}$ ) faces perpendicular to $c$. Microscopic examination of these faces following brief etching with water showed striations running along $b$ on one face of each crystal, while the other face remained smooth. Crystals of $\mathrm{LiHzS}$ and LiDzS were reetched following application of a large electric field along $c$, and again etched after applying a large field in the opposite direction. In all cases, the striations appeared on the same side as originally. ${ }^{13}$
The field of about $2500 \mathrm{~V} / \mathrm{cm}$ was much larger than the published ${ }^{1}$ coercive field of $320 \mathrm{~V} / \mathrm{cm}$. If domain reversal resulting in a chemically equivalent domain had occurred, subsequent etching would have caused grooves to form on the opposite side. Accordingly, reversal of chemically equivalent domains does not occur in LiHzS.

Chemically, inequivalent domains could in principle exist if, for example, domain reversal involved only reversal of the $\mathrm{N}-\mathrm{H} \cdot \mathrm{N}-\mathrm{N}$ dipole chains, with the $\mathrm{LiSO}_{4}{ }^{-}$framework remaining nearly fixed. But neutron diffraction results show that in $\mathrm{LiDzS}$ at least $95 \%$ of the deuterons in these dipole chains are in ordered positions relative to the framework. ${ }^{7}$ With $\mathrm{LiHzS}$ the minimum order may be somewhat lower because neutrons scatter incoherently from protons. Accordingly if such domains were to exist, the higher-energy domains could occupy only a small fraction of the volume. But then the hysteresis loops should be highly asymmetric, and also the ac susceptibility should be strongly dependent on the sign of an applied dc bias field. We have observed only very small asymmetries in these phenomena for $\mathrm{LiHzS}$. We conclude on the basis of the above considerations that this crystal is not ferroelectric.

This conclusion had been reached previously by Niizeki and Koizumi ${ }^{3}$ on the basis of structural considerations mentioned above and in view of the unpublished observation by Furuhata that the saturation polarization increase polarization increases with applied field. They suggested that the anomalous dielectric properties may be related to proton behavior within hydrazinium chains, and a kind of polarization relaxation suggested by Furuhata, but gave no further details of the mechanism. In the following discussion, we consider two nonferroelectric mechanisms, namely, (c) and (d) given above.

The $\mathrm{N}-\mathrm{H} \cdots \mathrm{N}-\mathrm{H} \cdots$ dipole chains have been shown by neutron diffraction studies to be nearly ordered, but one would expect a certain amount of dynamic disorder associated with hydrogen motion. Each chain can be approximated as a one-dimensional Ising chain with a nearest-neighbor interaction tending to align adjacent dipoles, and with a bias field representing the tendency of the noncentric structure to order the dipole chain. The ac susceptibility of such a biased one-dimensional Ising model has been derived recently by one of us. ${ }^{14}$ Because the audio-frequency ac susceptibility does not show the strong dependence on sign of dc bias field predicted for reversal of these dipoles, we conclude that the effective bias field is so large that this mechanism has little effect below radio frequencies. As will be discussed later, these dipoles may make a significant contribution to the susceptibility at high frequency.

We believe that the anomalous dielectric proper- 


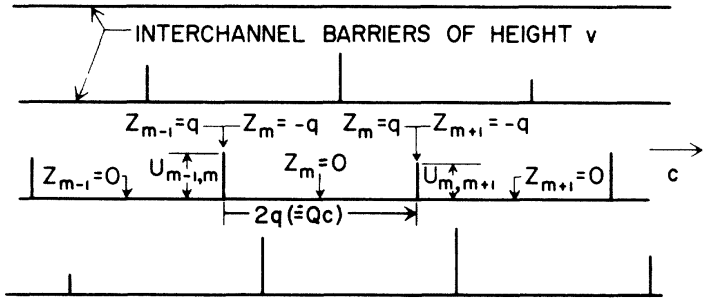

FIG. 3. Schematic of random barrier height model.

ties of LiHzS are caused by the fourth mechanism mentioned above, which is protonic conduction along the channels parallel to the $c$ axis, with extrinsic barriers of various heights partially blocking the flow. Our model for this mechanism is developed in Sec. II of this paper. In Sec. III we discuss our methods of measuring the dc conductivity and the dielectric susceptibility at audio, radio, and microwave frequencies. The results of these measurements are given in Sec. IV, and compared with predictions of our model in Sec. V. Our conclusions are presented in Sec. VI.

\section{PARTIALLY BLOCKED CHANNEL MODEL}

Both the real and imaginary components of the dielectric susceptibility of $\mathrm{LiHzS}$ are very large at high temperature and low frequency, with approximately exponential temperature dependence, and vary roughtly as $\omega^{-1 / 2}$ over several decades of frequency. This is in sharp contrast to the usual types of dielectric behavior. A ferroelectric has a very high dielectric susceptibility only near its transition temperature. Dipolar reorientation having a single relaxation time gives a characteristic step in the real component, and a peak in the imaginary component, of the susceptibility plotted against frequency, and it is difficult to imagine a reason for a distribution of relaxation times broad enough to yield our observed results. The usual types of frequencyindependent conduction mechanisms make a contribution only to the imaginary part of the susceptibility, which varies as $\omega^{-1}$. For "one-dimensional conductors" such as $\mathrm{LiHzS}$, however, we will show that impurity ions or other crystal defects which are commonly present can cause the observed dielectric behavior.

Localized barriers to flow, such as impurity ions, do not make much of a contribution to the real part of the dielectric susceptibility for isotropic conductors because the carriers can diffuse around the barriers. But if flow is restricted to channels running in one direction, barriers can block flow and give a significant contribution to the real part of the susceptibility.

In our model for this partially blocked flow, the carriers are excess or vacancy protons or deutrons having average density $N$ governed by an activation energy. A carrier can jump from a site arbitrarily chosen as the origin to six adjacent positions, two in the same hydrazinium ion channel at $\left(0,0, \pm \frac{1}{2} c\right)$ and four in adjacent channels at $\left(\frac{1}{2} a, \pm \frac{1}{2} b, 0\right)$ and $\left(-\frac{1}{2} a, \pm \frac{1}{2} b, 0\right)$. We assume that all jumps are thermally activated, with probability per unit for jumping over a barrier of height $U$ being $\nu_{0} e^{-\beta U}$, where $\beta=(k T)^{-1}$ and $\nu_{0} \simeq(\beta h)^{-1}$. We assume barriers of uniform height $v$ opposing jumps between channels as illustrated in Fig. 3, with lower barriers of uniform height $u_{0}$ opposing most jumps within a channel. The extrinsic barriers caused by impurity ions or other localized defects divide the channels into segments, with the barrier between the $m$ th and $(m+1)$ th segment having height $u_{m, m+1}+u_{0}$. Except for special cases we assume a distribution in $u_{m, m+1}$. The random variation in segment length is not so important, so we assume uniform distance $2 q$ between extrinsic barriers in a given channel. No correlation of extrinsic barrier locations in adjacent channels is assumed.

We consider first the conductivity perpendicular to the channels. Using the Einstein relation between mobility $\mu$ and diffusion constant $D$, and using for $D$ the usual expression for diffusion via jumps, noting that there are four neighboring sites having displacement $\pm \frac{1}{2} a$ in the $a$ direction, we obtain

$$
\begin{aligned}
& \sigma_{a}=N e \mu_{a}=N e^{2} \beta D_{a}=\frac{1}{2} N e^{2} \beta a^{2} \nu_{0} e^{-\beta \nu}, \\
& \sigma_{b}=\sigma_{a} b^{2} / a^{2} .
\end{aligned}
$$

The conductivity perpendicular to $c$ according to this model is not affected by the extrinsic barriers, is independent of frequency, and is slightly anisotropic.

With a time-dependent field applied along $c$, the barriers cause a position- and time-dependent fractional deviation $n$ in the carrier density, which then is expressed as $N(1+n)$. The current density $J$ within a given channel in a region between extrinsic barriers then can have both a conduction and a diffusion term:

$$
J=\sigma_{i} E-D_{i} e N \frac{\partial n}{\partial z}=\sigma_{i}\left(E-(e \beta)^{-1} \frac{\partial n}{\partial z}\right),
$$

where $\sigma_{i}$ is the intrinsic $c$-axis conductivity which would exist in the absence of the extrinsic barriers and the Einstein relation has again been used. This treatment of $n$ as a continuous function of position in the $z(c)$ direction is quite accurate if the number of possible carrier sites between extrinsic barriers is large. The barrier separation is $2 q$ while the site separation is $\frac{1}{2} c$, so there are $4 q / c-1$ sites between adjacent barriers. Implicit in Eq. (2) is the assumption that $n \ll 1$, so that $\sigma_{i}$ is essentially independent of position. For a large field this condition is not satisfied, and saturation effects occur. We consider now the flow across an extrinsic 
barrier, such as the $m, m+1$ barrier in Fig. 3 . This barrier is higher by an amount $u_{m, m+1}$ than the intrinsic barriers, and accordingly both terms in Eq. (2) are reduced by the factor $e^{-\beta u_{m}, m+1}$. For $N \partial n / \partial z$ we have the density difference

$$
N\left[n_{m}\left(q-\frac{1}{4} c\right)-n_{m+1}\left(-q+\frac{1}{4} c\right)\right] \simeq N\left[n_{m}(q)-n_{m+1}(-q)\right]
$$

for the nearest sites on opposite sides of the barrier, divided by their separation $\frac{1}{2} c$, so Eq. (2) becomes

$J_{m, m+1}=e^{-\beta u_{m, m+1}} \sigma_{i}\left\{E-2(c e \beta)^{-1}\left[n_{m}(q)-n_{m+1}(-q)\right]\right\}$

for current flow across the $m, m+1$ barrier.

The continuity equation contains for the rate of change of charge density within a given channel the usual term $-\partial J / \partial z$ and also a term describing flow to and from adjacent channels. The probability that a given site in a channel is occupied by a carrier is $\frac{1}{4} a b c N(1+n)$, while the probability that a given neighboring site in an adjacent channel is occupied is simply $\frac{1}{4} a b c N$ because extrinsic barrier locations in adjacent channels are assumed to be uncorrelated. With four adjacent channels, the net jump rate to adjacent channels is $a b c N n \nu_{0} e^{-\beta v}$, with a corresponding rate of change of density $-4 N n \nu_{0} e^{-\beta v}$. The continuity equation then becomes

$$
\frac{e N \partial n}{\partial t}=\frac{-\partial J}{\partial z}-4 e N n \nu_{0} e^{-\beta v}
$$

From Eq. (2),

$$
\frac{-\partial J}{\partial z}=D_{i} e N \frac{\partial^{2} n}{\partial z^{2}}
$$

and Eq. (4) becomes

$$
\frac{\partial n}{\partial t}=D_{i} \frac{\partial^{2} n}{\partial z^{2}}-4 n \nu_{0} e^{-\beta v} .
$$

For a small applied field $E=E_{0 z} e^{i \omega t}$ to which $n$ responds linearly, the general solution for Eq. (5) is

$$
n=\left(n_{s} \sinh k z+n_{c} \cosh k z\right) e^{i \omega t},
$$

where

$$
D_{i} k^{2}=i \omega+4 \nu_{0} e^{-\beta v}
$$

and $n_{s}$ and $n_{c}$ are complex arbitrary constants. One can attempt to express $k$ in terms of $\sigma_{i}$ and $\sigma_{a}$ by multiplying Eq. (7) by $N e^{2} \beta / \sigma_{i}$, using Eq. (1). In the result

$$
k^{2}=i \omega / D_{i}+8 \sigma_{a} / a^{2} \sigma_{i},
$$

the first term on the right can be evaluated only if $D_{i}$ (or equivalently $\mu_{i}$ ) is measured, or if in $D_{i}$ $=\frac{1}{4} c^{2} \nu_{0} e^{-\beta u_{0}}$ the activation energy $u_{0}$ is assumed to be zero, and one assumes some value such as $(\beta h)^{-1}$ for $\nu_{0}$.

To determine the arbitrary constants $n_{s}$ and $n_{c}$ we invoke continuity of current across the barriers in a given channel. From Eq. (2) the current den- sity within the $m$ th segment is

$$
J_{m}=\sigma_{i}\left(E-\frac{k\left(n_{s m} \cosh k z+n_{c m} \sinh k z\right) e^{i \omega t}}{e \beta}\right),
$$

using $n$ from Eq. (6). For the $m, m+1$ barrier, located at $z_{m}=q$ or, equivalently, at $z_{m+1}=-q$, continuity requires that $J_{m}(q)=J_{m+1}(-q)$ or $n_{s m} \cosh k q+n_{c m} \sinh k q=n_{s, m+1} \cosh k q-n_{c, m+1} \sinh k q$.

Also, continuity requires that $J_{m}(q)$ be equal to $J_{m, m+1}$ as given in Eq. (3), so

$$
\begin{aligned}
& 2 e^{-\beta u_{m}, m+1}\left(n_{s m} \sinh k q+n_{c m} \cosh k q\right. \\
& \left.+n_{s, m+1} \sinh k q-n_{c, m+1} \cosh k q\right) \\
& +k c\left(n_{s m} \cosh k q+n_{c m} \sinh k q\right) \\
& =\beta e E_{0 z} c\left(1-e^{-\beta u_{m}, m+1}\right) \cdot \quad \text { (11) }
\end{aligned}
$$

The arbitrary constants can be obtained from Eqs.

(10) and (11) and the boundary conditions, as will be illustrated for several special cases.

The contribution $\chi_{c j}$ of this conduction mechanism to the $c$-axis ac dielectric susceptibility is related to the current density $J$ by

$$
\begin{aligned}
\chi_{c j} & =\chi_{c j}^{\prime}-i \chi_{c j}^{\prime \prime}=\frac{\int P d V}{V E}=\frac{\iint J d t d V}{V E}=\frac{\int J d V}{i \omega V E} \\
& =\frac{\left\langle\int_{-q}^{q} J_{m} d z_{m}\right\rangle_{\mathrm{av}}}{2 i \omega q E}=\frac{\sigma_{i}}{i \omega}\left(1-\frac{\left\langle n_{s}\right\rangle_{\mathrm{av}} \sinh k q}{q E_{0 z} e \beta}\right),
\end{aligned}
$$

where the averages are taken over all the segments, which are $V / a b q$ in number.

Several special cases are now presented to provide a qualitative feeling for the phenomena which can occur. The first case has extrinsic barriers of uniform height $u+u_{0}$. Then symmetry requires that $n_{s m}=n_{s, m+1} \equiv n_{s}$ and $n_{c m}=n_{c, m+1} \equiv n_{c}$, and it follows from Eq. (10) that $n_{c}=0$. Solution of Eq. (11) then yields

$$
\left\langle n_{s}\right\rangle_{\mathrm{av}}=n_{s}=\frac{\beta e E_{0 z} c\left(1-e^{-\beta u}\right)}{4 e^{-\beta u} \sinh k q+k c \cosh k q},
$$

which is inserted into Eq. (12) to give

$$
\chi_{c j}=\frac{\sigma_{i}}{i \omega}\left(1-\frac{1-e^{-\beta u}}{k q \operatorname{coth} k q+4 q c^{-1} e^{-\beta u}}\right) .
$$

It is seen that at high frequencies for which $k q \gg 1$, the extrinsic barriers lose their effect, and the material acts as an ordinary conductor of conductivity $\sigma_{i}$. From Eq. (8), this occurs for $\omega \gg D_{i} / q^{2}$. The crystal will act as an intrinsic conductor at all frequencies if the extrinsic barriers are low enough and/or widely separated enough so that $e^{-\beta u} \simeq 1$ and/ or $4 q c^{-1} e^{-\beta u} \gg 1$. Conversely, if $4 q c^{-1} e^{-\beta u} \ll 1$ and $k q \ll 1$, then $\chi_{c j} \ll \sigma_{i} / i \omega$ and Eq. (14) can be expanded to yield

$$
\chi_{c j} \simeq \frac{\sigma_{i} q^{2}}{3 D_{i}}+\left(\frac{1+4 q}{c}\right) e^{-\beta u} \frac{\sigma_{i}}{i \omega}+\frac{8 q^{2}}{3 a^{2}} \frac{\sigma_{a}}{i \omega} .
$$




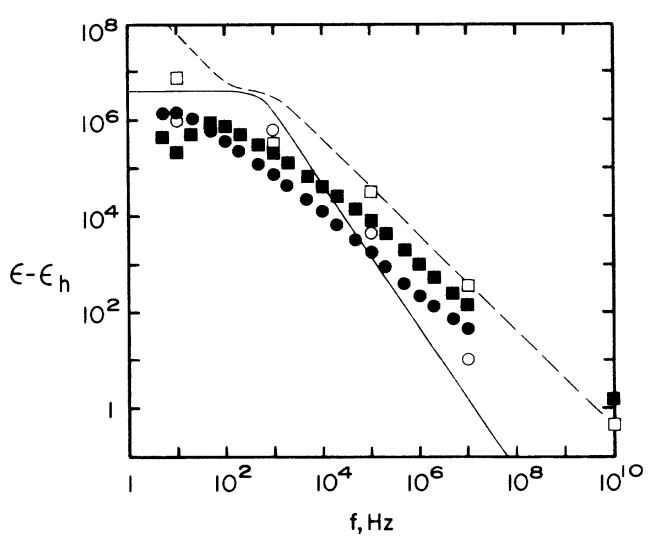

FIG. 4. Conductivity contribution to the dielectric constant vs frequency, at $10^{3} / T\left({ }^{\circ} \mathrm{K}\right)=2.1\left(476^{\circ} \mathrm{K}\right)$. Solid symbols are experimental points for LiHzS No. 6 (except another crystal at $9.33 \mathrm{GHz}$ ). Open symbols are from random barrier height model for $E_{1}=E_{N} / 10^{3} k=5.5, E_{2}$ $=v / 10^{3} k=12$, and $Q=2 q / c=100$. Lines are for model having all barriers of equal height $U=u / 10^{3} k=6.86$, with $E_{1}=5.5, E_{2}=12$, and $Q_{u}=73,000$ (energies expressed in units of $\left.1000^{\circ} \mathrm{K}\right)$. Circles and solid line represent $\epsilon^{\prime}$, squares and dashed line represent $\epsilon^{\prime \prime}$.

Under these conditions the $c$-axis conductivity $i \omega \chi_{c}^{\prime \prime}$ is the product of $i \omega$ and the last two terms of Eq. (15). If the last term dominates, the carriers bypass the extrinsic barriers by flowing to adjacent channels. The temperature dependence is the same as for $\sigma_{a}$ and $\sigma_{b}$, but

$$
\sigma_{c} / \sigma_{a}=8 q^{2} / 3 a^{2} \gg 1 \text { for } q \gg c,
$$

and the conductivity is highly anisotropic. If for the same large $q / c$ ratio the $\sigma_{i}$ term in the conductivity dominates, the conductivity will be even more anisotropic.

The frequency dependence of the real and imaginary components of that part of the dielectric constant related to the partially blocked conductivity is shown in Fig. 4, where $\epsilon-\epsilon_{h}=\chi_{c j} / \epsilon_{0}$ in mks units. At low and high frequencies the imaginary or conductive component dominates. At intermediate frequencies the real or capacitive component related to charge pileup against extrinsic barriers becomes comparable in size. For other choices of parameters it can dominate in the middle-frequency range, while the imaginary component will have a positive slope of unity in this range.

Further discussion of Fig. 4 appears in Sec. V. Expressions for the susceptibility for other simple repetitive sequences of barrier heights are given in the Appendix.

For the general case of random barrier heights, the actual distribution of heights is unknown, so we have chosen a plausible model. We consider extrinsic barriers caused by impurities of positive charge $e$ having random spatial distribution. Their density as determined by comparing this theory with experiment is greater than the carrier density, so that screening effects from other carriers can be neglected. The impurities lie at varying perpendicular distances from a given channel, but are assumed to form barriers to flow of positive carriers at equal intervals $2 q$ along the channel. The maximum interaction energy between impurity and carrier is $e^{2} / 4 \pi \epsilon_{0} \epsilon_{h} r$ in mks units, with $\epsilon_{h}$ being the high-frequency dielectric constant. For small $r$ the barrier height $u$ will just be this interaction energy. As $r$ increases the Coulomb fields overlap, so that if $r=q / \pi$ for all impurities the barriers are only about half as high as for an isolated impurity at the same radius. We make the approximation that $u$ is given by

$$
\begin{array}{ll}
u=e^{2} / 4 \pi \epsilon_{0} \epsilon_{h} r & (r \leq q / \pi), \\
u=0 & (r>q / \pi) .
\end{array}
$$

The smallest value of $u$ then is $u_{s}=e^{2} / 4 \epsilon_{0} \epsilon_{h} q$.

The dielectric behavior depends not only on the energy distribution of the barriers but also on their spatial distribution along a given channel. For instance, if all the high barriers are in one region and the low barriers in another, the susceptibility will be greater than if the barriers have a random spatial distribution. For this reason it appears difficult to obtain an analytic expression for the susceptibility for this model. Instead we have calculated the susceptibility with a computer program which generates barrier height sequences from random numbers $w$ uniformly distributed between 0 and 1 using the relation $u=u_{s} w^{-1 / 2}$. To demonstrate the correctness of this relation we show that for a volume $d V$ containing impurity ions giving rise to energy barriers in the range corresponding to $d w$, the ratio $d V / d w$ is a constant independent of $w$ as required for random impurity distribution. Using $u=q u_{s} / \pi r$, we have

$$
d w=-\frac{2 w^{3 / 2} d u}{u_{s}}=-\frac{2 u^{2}}{u^{3}} \frac{d u}{d r} d r=\frac{2 \pi^{2} r d r}{q^{2}}=\frac{d V}{V},
$$

where $V$ is the volume $2 q^{3} / \pi$ of a cylinder of radius $q / \pi$ and length $2 q$ which contains on the average one impurity ion.

The susceptibility program requires three arbitrary input parameters in addition to frequency and temperature. These are $Q=2 q / c, E_{1}=E_{N} / 10^{3} k_{b}$, and $E_{2}=v / 10^{3} k_{b}$, where $E_{N}$ is the activation energy for carrier creation, $v$ is the activation energy for interchannel jumping, and $k_{b}$ is Boltzmann's constant. The parameters $\sigma_{i}, D_{i}$, and $\nu_{0}$ needed in Eqs. (7)-(12) were chosen by assuming $\nu_{0}=(\beta h)^{-1}$ and $\sigma_{i}=N e^{2} D_{i}$, with

$$
N=N_{0} e^{-E_{N}}=4 e^{-E_{N}} / a b c \text { and } D_{i}=\frac{1}{4} \nu_{0} c^{2} .
$$




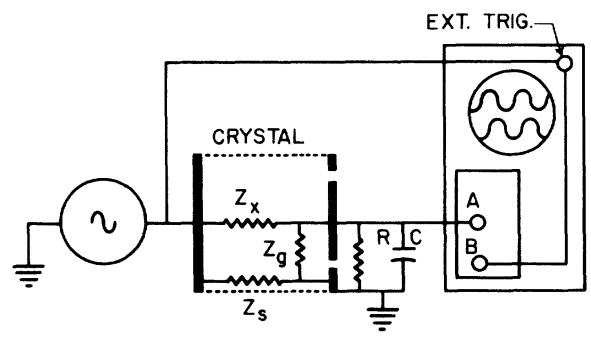

FIG. 5. Voltage divider circuit for measuring dielectric constant.

This $N_{0}$ corresponds to one potential carrier (the $\mathrm{N}-\mathrm{H} \cdots \mathrm{N}$ hydrogen) per molecule, while $D_{i}$ is assumed to have zero activation energy $u_{0}$. For a given temperature and frequency, the susceptibility is calculated for each of 50 barrier sequences, each sequence consisting of 199 barriers of random height. The mean susceptibility and its standard deviation are calculated for the group of 50 sequences. For the first sequence an arbitrary boundary condition of zero input and output current is applied. Then $n_{c 1}$ is found from Eq. (9) from the condition $J_{1}=0$ at $z_{1}=-q$. Equations (10) and (11) are then used to find $n_{s 1}$ in terms of $n_{s 2}, n_{s 2}$ in terms of $n_{s 3}$, etc. Finally, $n_{s 200}$ is calculated from the condition $J_{200}=0$ at $z_{200}=q$, its value is used to determine $n_{s 199}$, etc. The susceptibility for the sequence is then found using Eq. (12). Especially at low frequency the zero boundary currents artificially cause the susceptibility values to be too low, so for the second sequence of 199 barriers the boundary condition is that the current in and out is the average current from the first sequence as found from Eq. (12). For each succeeding sequence, the boundary current is the average current from the preceding sequence. The error in the average susceptibility caused by the low values from the first few sequences is negligible.

\section{EXPERIMENTAL METHODS}

Single crystals of $\mathrm{LiN}_{2} \mathrm{H}_{5} \mathrm{SO}_{4}$ were prepared by dissolving stoichiometric weights of reagent grade $\mathrm{Li}_{2} \mathrm{CO}_{3}$ and $\left(\mathrm{NH}_{2}\right)_{2} \cdot \mathrm{H}_{2} \mathrm{SO}_{4}$ in water, and crystallizing by slow evaporation. Crystals as large as $3 \times 5 \times 5$ $\mathrm{cm}$ with well-developed (100), (110), and (101) faces were grown. To obtain deuterated crystals, $\mathrm{LiN}_{2} \mathrm{H}_{5} \mathrm{SO}_{4}$ crystals were dissolved in $90 \% \mathrm{D}_{2} \mathrm{O}$, recrystallized by evaporation, then dissolved in $99.8 \%$ $\mathrm{D}_{2} \mathrm{O}$ and recrystallized. This recrystallization was performed in a desiccator to minimize water vapor exchange with the atmosphere. The atomic percentages of deuteration for two of these final $\mathrm{LiN}_{2} \mathrm{D}_{5} \mathrm{SO}_{4}$ crystals were determined by mass spectroscopy to be 81 and $84 \% .{ }^{15}$ In another mass spectroscopic study ${ }^{16}$ made for one of our undeuterated seed crystals, upper limits for impurity concentrations ex- pressed in parts per thousand were found to be $\mathrm{Na}: 1.5 ; \mathrm{Al}: 0.05 ; \mathrm{Si}: 0.02 ; \mathrm{Cl}: 4 ; \mathrm{K}: 2 ; \mathrm{C}: 2$; $\mathrm{Mn}: 0.02$; and $\mathrm{Fe}: 0.05$.

Samples for dc conductivity measurements and for audio- and radio-frequency dielectric susceptibility studies were prepared by using a wetted string saw to cut slices 1-2 mm thick with faces perpendicular to a crystal axis. These faces were then polished on wet paper, after which conducting silver paint contacts were applied. One face of each crystal was completely painted to provide the input contact, while the other face had a circular center contact separated by a narrow unpainted circle from a guard ring.

The equivalent circuit for the dc conductivity measurements and some of the ac measurements is shown in Fig. 5, except that for the dc studies a dc supply and an electrometer were used. The crystal is symbolized by three impedances, with $R_{g}$ being the real part of the impedance between input contact and guard ring, $R_{x}$ between input and center contacts, and $R_{s}$ between center contact and guard ring. The guard ring prevents surface current from flowing through the electrometer resistor $R$. One can check whether the desired conditions $R \ll R_{s}$ and $R \ll R_{x}$ are satisfied by reducing $R$ and noting whether the electrometer voltage drops by the same factor.

To prevent moisture from condensing on the crystal during measurements made below room temperature, the area around the crystal was bathed with dry nitrogen gas. The crystal and a chromelalumel thermocouple were within a copper container which ensured uniform temperature within the enclosure once equilibrium was reached. The exterior of the container was in contact with a bath at the desired temperature. The thermocouple output provided the $X$ voltage for a Moseley 7000 AR $X Y$ recorder. The electrometer output provided the $Y$ voltage, so the recorder trace provided a continuous measurement of the crystal current versus temperature. A problem with dc conductivity measurements on protonic conductors having electronically conducting electrodes is electrochemical decomposition at the anode, or "polarization." To minimize this effect, the current was kept as small as possible and the polarity of the applied voltage was periodically reversed. A large transient current was observed after each reversal, and the average current after the decay of the transient was taken to be indicative of the crystal conductivity.

The ac dielectric susceptibility measurements were made on LiHzS crystals Nos. 5 and 6 and LiDzS crystal No. 7 (the crystals with smaller numbers had been used in the conductivity studies). The sample holder was similar to that used in the conductivity measurements. The $c$-axis ac dielectric sus- 


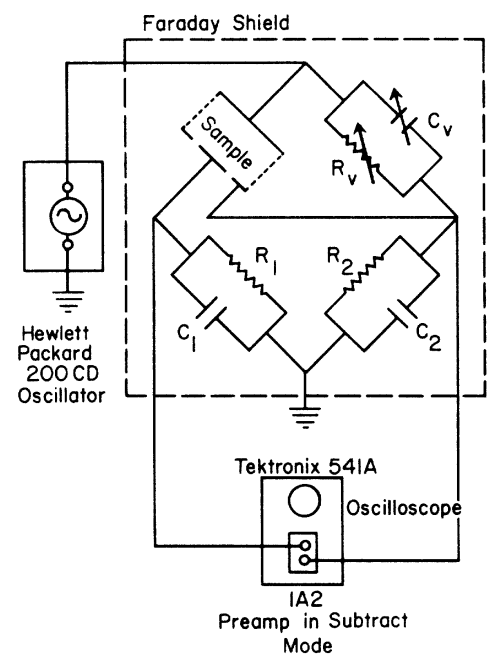

FIG. 6. Bridge circuit for measuring dielectric constant.

ceptibility for $\mathrm{LiN}_{2} \mathrm{D}_{5} \mathrm{SO}_{4}$ crystal No. 7 from $5 \mathrm{~Hz}$ to $100 \mathrm{kHz}$ and for $\mathrm{LiN}_{2} \mathrm{H}_{5} \mathrm{SO}_{4}$ crystal No. 5 was measured with the bridge circuit shown in Fig. 6. Here $C_{1}$ and $R_{1}$ are the input impedance of the $\times 10$ oscilloscope probe in parallel with the capacitance of the sample holder and a $100-\mathrm{pF}$ capacitor which was added to reduce the percentage uncertainty in $C_{1}$. The impedance level of the right half of the bridge is 1000 times less than that of the left half, so that the crystal impedance can be balanced by resistance $R_{v}$ and capacitance $C_{v}$ of magnitudes conveniently attainable using standard decade boxes. With the bridge balanced, the potentials of center contact and guard ring are equal, so the impedance between these contacts does not affect the balance conditions. The current from input contact to guard ring is in parallel with the current through $R_{v}$ and $C_{v}$, but is generally much smaller, so to sufficient accuracy it can be assumed that the input contact to center contact impedance can be represented by a resistor $R_{x}=100 R_{v}$ in parallel with a capacitor $C_{x}$ $=C_{v} / 1000$. In mks units the relative dielectric constant $\epsilon$ is given by

$$
\epsilon=\epsilon^{\prime}-i \epsilon^{\prime \prime}=\left(d / \epsilon_{0} A\right)\left(C_{x}-i \omega R_{x}\right),
$$

and the susceptibility is given by $\chi=\chi^{\prime}-i \chi^{\prime \prime}$ $=\epsilon_{0}(\epsilon-1)$, where $d$ and $A$ are the crystal thickness and effective center contact area. This bridge method was quite sensitive, since it was possible to null out the fundamental frequency almost completely by adjusting $R_{v}$ and $C_{v}$ while observing the oscilloscope. This method was used for frequencies ranging from the $5-\mathrm{Hz}$ lower limit of the audio oscillator up to $100 \mathrm{kHz}$. Above this frequency the inductance in the decade boxes made results unreliable. Additional details concerning the foregoing experimental methods appear elsewhere. ${ }^{17}$
The remaining audio- and radio-frequency susceptibility measurements were made using the circuit in Fig. 5. For the $a$ - and $b$-axis measurements and for the $c$-axis measurements above 500 $\mathrm{kHz}$ the crystal impedance was quite large, and to get a suitable large signal on the oscilloscope, it was necessary to make the parallel impedance of $R$ and $C$ as large as possible while keeping it small compared to $Z_{g}$. For $a$ - and $b$-axis measurements, the low $c$-axis impedance made $Z_{g}$ quite small, and it would have been preferable to keep the guard ring at the center contact potential with a suitable circuit. With the oscilloscope probe in parallel with the sample holder, $R$ was $10 \mathrm{M} \Omega$ and $C$ was $30.4 \mathrm{pF}$. For LiHzS crystal No. 6, $C$ was increased for certain measurements made at high temperature because of the reduced crystal impedance. A computer program was used to determine $\epsilon^{\prime}$ and $\epsilon^{\prime \prime}$ from the crystal dimensions and the measured values of $V_{a}, V_{b}$, and $\phi_{b}-\phi_{a}$. This voltage divider method does not have the inherent accuracy of the bridge method, because it is limited to the accuracy to which voltages and phase differences can be observed on the oscilloscope face. It was employed to obtain data at higher frequencies, and gave reliable results up to about $10 \mathrm{MHz}$.

The microwave dielectric constant measurements were made using a standard shorted line technique ${ }^{18}$ and were taken at a frequency of $9.330 \mathrm{GHz}$. As reported earlier, ${ }^{19}$ this method was chosen ${ }^{20}$ as being simplest to use since it is possible to grow rather large crystals of good quality and to cut and polish them to suitable shape. The crystals were polished to convenient thicknesses and to a cross section which completely filled the waveguide, with the axis being measured parallel to the electric field direction in the waveguide. They were mounted against a shorting plate made of copper for better heat transfer to the thermal bath. A heater coil surrounded a portion of the stainless-steel waveguide and end plate with the whole assembly placed in an evacuating chamber which in turn was mounted in a Dewar system.

Measurements between liquid-nitrogen and room temperatures were made by first cooling, then taking data either as the system warmed naturally, which took many hours, or by using the heating coil. Consistent results were attained either way but it was necessary to partially evacuate and backfill the sample chamber with dry nitrogen gas to reduce moisture condensation which would lead to high losses. The heating coil allowed temperatures up to crystal destruction to be easily attained. Measurements at any particular temperature required waiting for the crystal to come to equilibrium which usually took about 10 min for samples that filled the $X$-band waveguide and were less than $\frac{1}{2} \mathrm{~cm}$ thick. Temperature measurements were made with 


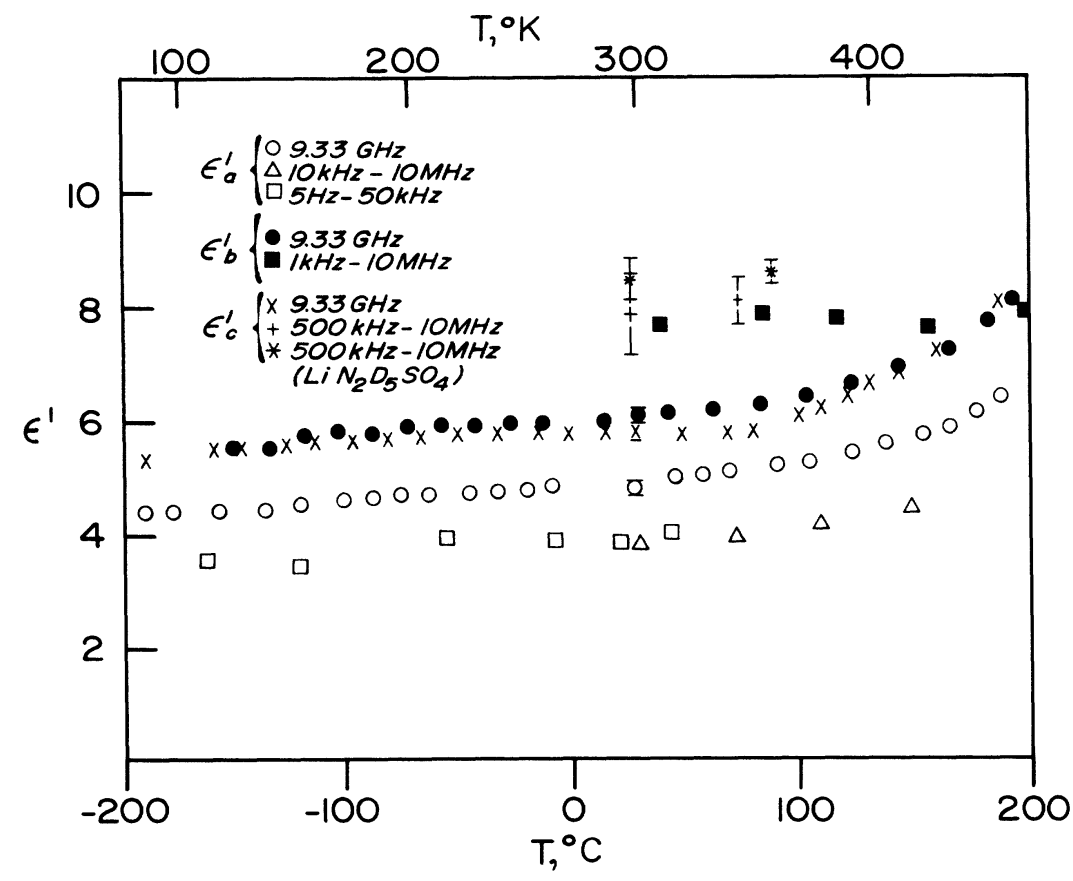

Fig. 7. Temperature dependence of real part of "high-frequency dielectric constant" $\epsilon_{h}$, obtained at combinations of temperature and frequency for which the conduction mechanism makes no appreciable contribution. Results are for $\mathrm{LiN}_{2} \mathrm{H}_{5} \mathrm{SO}_{4}$ except as noted. a thermocouple in direct contact with the crystal.

\section{EXPERIMENTAL RESULTS}

At audio and radio frequencies, $\epsilon$ contains a contribution $\epsilon-\epsilon_{h}$ from the conduction mechanism and a contribution $\epsilon_{h}$ from other mechanisms which relax at higher frequencies. To separate these contributions, we determined $\epsilon_{h}$ from measurements made at $9.330 \mathrm{MHz}$, because the conduction mechanism has little effect at such high frequency. The real and imaginary parts $\epsilon^{\prime}$ and $\epsilon^{\prime \prime}$ of the dielectric constant at $9.330 \mathrm{MHz}$ as well as the loss tangent $\left(\tan \delta=\epsilon^{\prime \prime} / \epsilon^{\prime}\right)$ for $\mathrm{LiN}_{2} \mathrm{H}_{4} \mathrm{SO}_{4}$ at room temperature are given in Table I. The dependence on temperature of the real part of the dielectric constant and the loss tangent are plotted in Figs. 7 and 8 . The error bars shown on both figures include measurement and observational errors only. Specifically they include the error owing to temperature expansion of the crystal, ${ }^{21}$ which amounts to less than the typical accuracy in thickness for a water-polished crystal, and for apparent changes in observation owing to thermal expansion of the waveguide. The error bars do not include variations in measurement as a function of crystal history. It was noted, for example, that excessive heating and cooling caused significant clouding of the crystal which in turn produced different values of $\epsilon^{\prime}$ particularly. The cloudiness is due to crystal deterioration but quantitative results for degree of decomposition for different runs are not available. To account for this uncertainty, the room-temperature error bars should be about twice as large.
In at least one case and after a certain amount of this cloudiness had occurred, a definite resonant behavior of the real and imaginary parts of $\epsilon$ was observed for temperatures near $+137^{\circ} \mathrm{C}$.

The microwave measurements show an increase of $\epsilon^{\prime}$ with temperature which becomes quite rapid at high temperature. This rapid increase may be associated with the structure change which causes the change in proton and lithium NMR line shape and spectra above $120^{\circ} \mathrm{C}{ }^{8}$ The values of $\epsilon_{a}^{\prime}, \epsilon_{b}^{\prime}$, and $\epsilon_{c}^{\prime}$ differ somewhat, as expected for an orthorhombic unit cell.

For certain temperature-frequency combinations at radio and audio frequencies, the conduction mechanism apparently does not make an appreciable contribution to $\epsilon^{\prime}$; these values of $\epsilon^{\prime}$ are shown in Fig. 7 also. The measurements of $\epsilon_{a}$ at low frequency were made using a bridge method at lower temperature and a voltage divider method at higher temperature. The values of $\epsilon_{a}^{\prime}$ from the two methods agree well, but are lower than the $9.330-\mathrm{MHz}$ values. One expects the microwave value to be no larger than the low-frequency value, so it is possible that an unknown systematic error causes this discrepan-

TABLE 1. Dielectric constants and loss tangents at room temperature.

\begin{tabular}{llll}
\hline Axis & \multicolumn{1}{c}{$\epsilon^{\prime} / \epsilon_{0}$} & \multicolumn{1}{c}{$\epsilon^{\prime \prime}$} & \multicolumn{1}{c}{$\tan \delta$} \\
\hline$a$ & $4.80 \pm 0.20$ & $0.043 \pm 0.030$ & $0.0089 \pm 0.0040$ \\
$b$ & 6.11 & 0.048 & 0.0078 \\
$c$ & 5.89 & 0.052 & 0.0088 \\
\hline
\end{tabular}




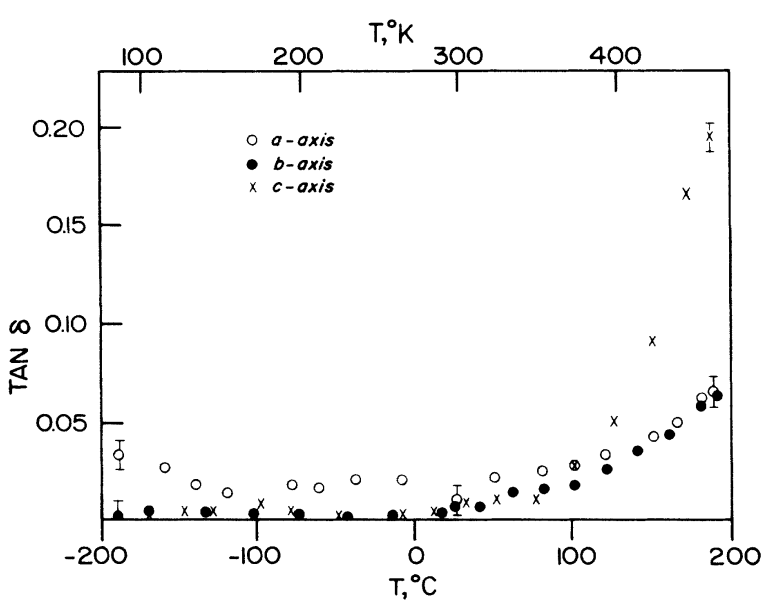

FIG. 8. Temperature dependence of loss tangent $\epsilon^{\prime \prime} / \epsilon^{\prime}$ at $9.330 \mathrm{GHz}$.

cy. For $\epsilon_{b}^{\prime}$ and $\epsilon_{c}^{\prime}$, however, the low-frequency values are larger than the microwave values. The difference in $\epsilon_{c}^{\prime}$ may be caused by dynamic disorder in the $\mathrm{N}-\mathrm{H} \cdots \mathrm{N}-\mathrm{H}$ chains as discussed in Sec. I.

According to our theory, for random extrinsic barriers, $\sigma_{c}$ should approach exponential temperature dependence at high frequency, with the effective activation energy decreasing until at very high frequency the extrinsic barriers become ineffective and the activation energy is that for $\sigma_{i}$. An upper limit for this activation energy for $\sigma_{i}$ is obtained from the microwave frequency loss tangent shown in Fig. 8. The rapid increase in $\tan \delta_{c}$ above $100^{\circ} \mathrm{C}$ corresponds to an activation energy of $0.45 \pm 0.05$ $\mathrm{eV}$. The dc and ac conductivity results presented in Fig. 9 show this increase in $\sigma_{c}$ and decrease in activation energy with increasing frequency. A hypothetical LiHzS crystal without extrinsic barriers would by this interpretation have a dc conductivity several orders of magnitude larger than for any other reported single-crystal protonic semiconductor.

The $a$ - and $b$-axis conductivities shown in Fig. 9 are of interest because they give information on the interchannel barriers which effect $\sigma_{c}$. We find lower dc conductivity than reported by Vanderkooy, Cuthbert, and Petch, ${ }^{2}$ but we agree with their findings that $\sigma_{a}$ and $\sigma_{b}$ are much smaller than $\sigma_{c}$ at room temperature while at higher temperature the activation energies for $\sigma_{a}$ and $\sigma_{b}$ increase so that $\sigma$ is almost isotropic above $200^{\circ} \mathrm{C}$. The preexponential factor $\sigma_{0 a}$ is abnormally small at lower temperature and abnormally large at higher temperature, in comparison with $\sigma_{0 a}=2 e^{2} a / b c h$ obtained from Eq. (1) in the infinite-temperature limit using $N_{0}=4 / a b c$ and $\nu_{0}=1 / \beta h$. Similar anomalous behavior is found for $\sigma_{0 b}$, while $\sigma_{0 c}$ for the $9.33-\mathrm{MHz}$ results in Fig. 9 exhibits the order-of-magnitude agreement with the calculated value which is more typical for protonic semiconductors.

Low values of $\sigma_{0}$ usually are attributed to extrinsic carriers produced by donors or acceptors, but in $\mathrm{LiHzS}$ the possibility also exists that at lower temperatures the $a$ - and $b$-axis mobilities are determined by abnormally low interchannel barriers associated with permanent defects or impurities. Additional low barriers produced thermally by a reversible process could cause the large activation energy and $\sigma_{0}$ observed at higher temperature. A possible reversible process is related to the previously reported ${ }^{1,2}$ surface decomposition. Recent studies $^{22}$ show that this surface decomposition has an activation energy of $1.35 \mathrm{eV}$, with $(d m / d t) / m$ at $210^{\circ} \mathrm{C}$ of about $0.016 / \mathrm{h}$ for powder having estimated grain diameter of 10 to $100 \mu$. The decomposition produces an $\mathrm{H}_{2} \mathrm{~S}$ smell and leaves a lithium sulfate residue, indicating the reaction

$$
2 \mathrm{LiN}_{2} \mathrm{H}_{5} \mathrm{SO}_{4}-\mathrm{Li}_{2} \mathrm{SO}_{4}+\mathrm{H}_{2} \mathrm{~S}+4 \mathrm{H}_{2} \mathrm{O}+2 \mathrm{~N}_{2} \text {. }
$$

If this reaction occurs reversibly in the interior of the crystal with activation energy $E_{d}$ and healing time $\tau_{h}$, then in equilibrium the decomposition rate $N_{0} \nu_{0} e^{-\beta E_{d}}$ is equal to the healing rate $N_{d} / \tau_{h}$, giving defect concentration $N_{d}=N_{0} \nu_{0} \tau_{h} e^{-\beta E_{d}}$. If $N_{d} / N_{0}$ is assumed equal to unity at the bulk decomposition temperature $T_{d}$, then $\nu_{0} \tau_{h}=e^{E_{d^{\prime}} k T_{d}}$. If the missing sulfate ions destroyed in this reaction provide zero-

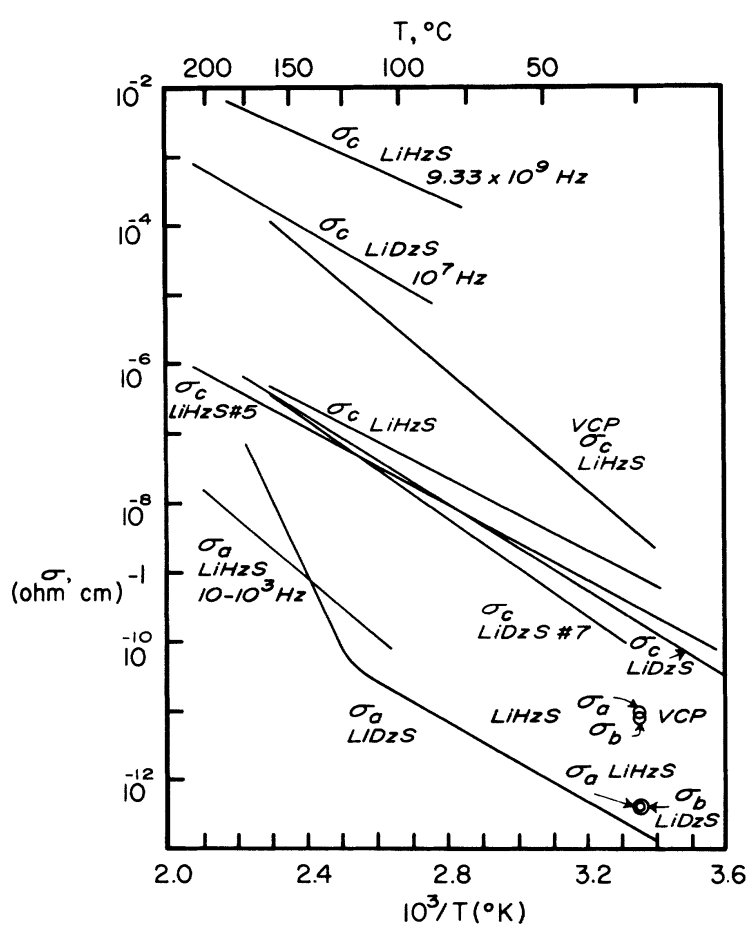

FIG. 9. Conductivity of LiHzS and LiDzS along crystallographic axes. VCP represents data obtained by Vanderkooy, Cuthbert, and Petch (Ref. 2). 


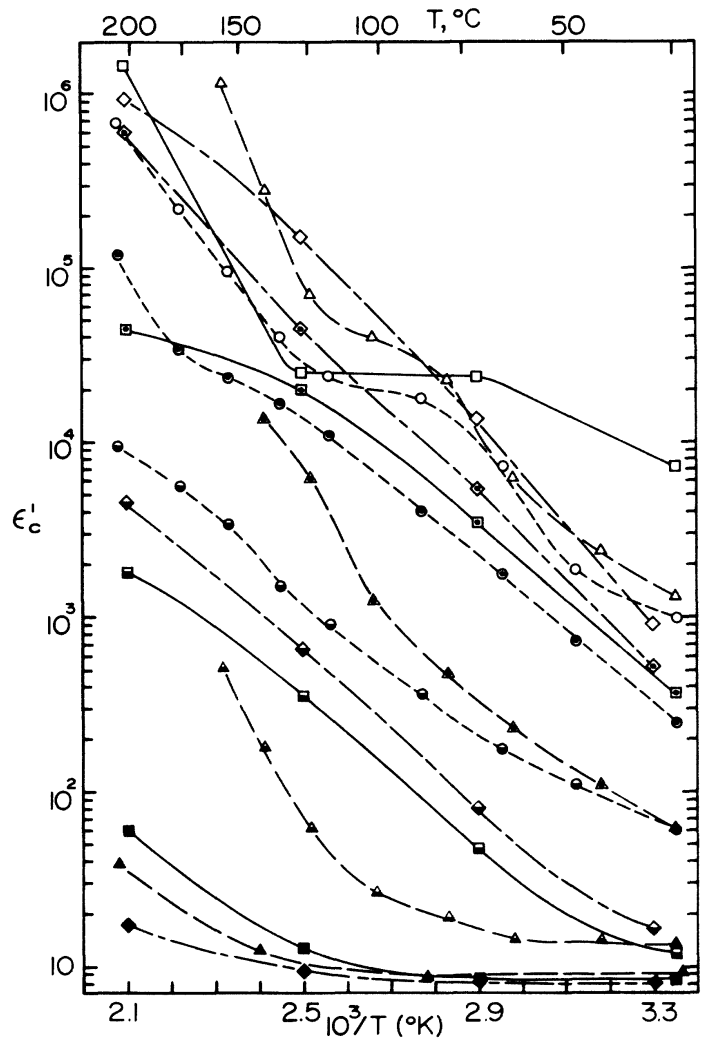

FIG. 10. Temperature dependence of real part of $c^{-}$ axis dielectric constant at $10 \mathrm{~Hz}$ (open symbols), $1 \mathrm{kHz}$ (symbols with dots), $100 \mathrm{kHz}$ (half-filled symbols), and $10 \mathrm{MHz}$ (filled symbols). Circles represent experimental points for LiHzS No. 5, squares for $\mathrm{LiHzS}$ No. 6, triangles for LiDzS No. 7, and diamonds represent points calculated using the random barrier height program for $Q=100, E_{1}=5.5$, and $E_{2}=12$.

activation-energy interchannel paths, then in Eq. (1) $e^{-\beta v}-e^{-\beta v}+\nu_{0} \tau_{h} e^{-\beta E_{d}}$. The second term would give an anomalously large $\sigma_{0}$ and could be responsible for the steep portion of the $\mathrm{LiDzS} \sigma_{a}$ curve in Fig. 9. The decomposition process is being studied in detail to resolve this question.

Only limited $a$ - and $b$ - axis ac results were obtained, because of the relatively high impedance in conjunction with the low $c$-axis shunt impedance between guard ring and ground (see Fig. 5). In general $\epsilon$ " dominated at high temperature and low frequency, while $\epsilon^{\prime}$ was larger under the opposite conditions.

The $c$-axis dielectric behavior is illustrated in Figs. 10-15. In Figs. 10 and 11 the temperature dependence is illustrated. The considerable variation from one crystal to another is evident, and this variation apparently is as large as any difference in behavior between deuterated and normal crystals. There is good qualitative agreement between the experimental points and the calculated points for a given set of the dimensionless parameters $E_{1}, E_{2}$, and $Q$, defined in Sec. II. Neither the calculated nor experimental points fall exactly in straight lines as would be predicted if the process were governed only by an activation energy. The experimental results at low frequency show quite pronounced humps which may correspond to peaks in the actual barrier height distribution. In these figures, the data for LiHzS No. 5 and for LiDzS No. 7 were obtained with the bridge circuit, except that the voltage divider circuit was used at frequencies above $10 \mathrm{kHz}$ with $\mathrm{LiDzS}$ No. 7. All data for LiHzS No. 6 were obtained with the voltage divider circuit. Other crystals were used for the microwave measurements.

The frequency dependence of the dielectric constant is shown in Figs. 12-14 for the three crystals studied at audio and radio frequencies. In Fig. 12 the microwave results are shown also. On these logarithmic plots a frequency-independent conductivity $\sigma$ would according to the relation $\sigma=\epsilon_{0} \omega \epsilon^{\prime \prime}$ give slope of -1 for the dashed lines representing $\epsilon^{\prime \prime}$. In general in the intermediate frequency region of $10 \mathrm{~Hz}$ to $100 \mathrm{kHz}$ the real and imaginary parts of $\epsilon$ are roughly equal and have slopes between 0 and

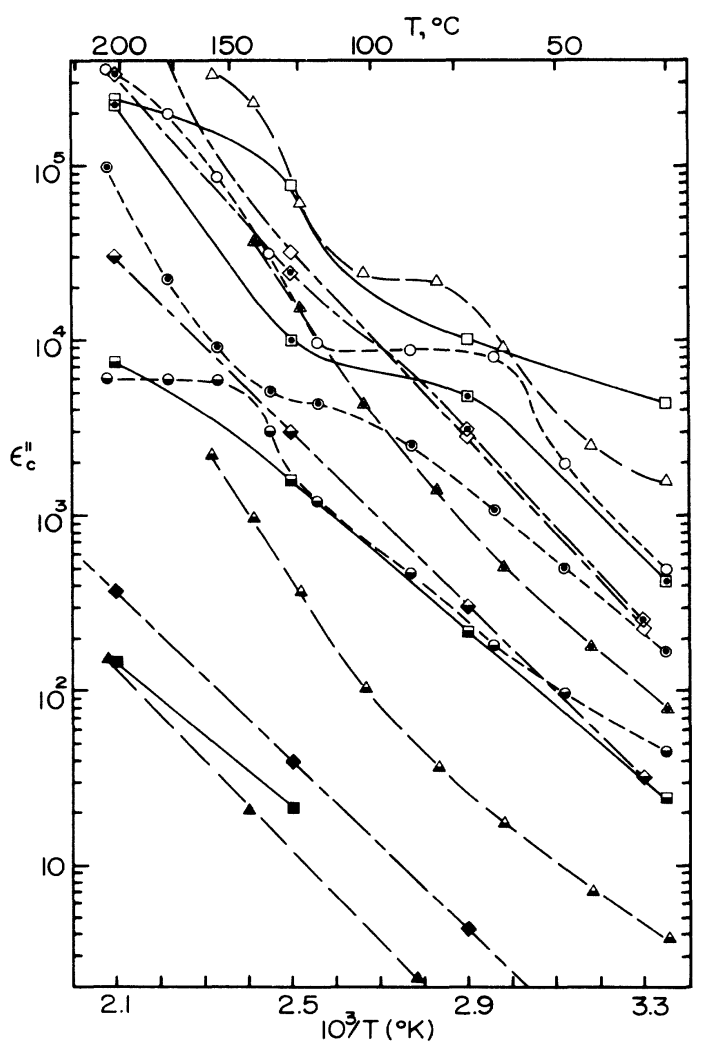

FIG. 11. Temperature dependence of imaginary part of $c$-axis dielectric constant. Points are labeled as in Fig. 10. 


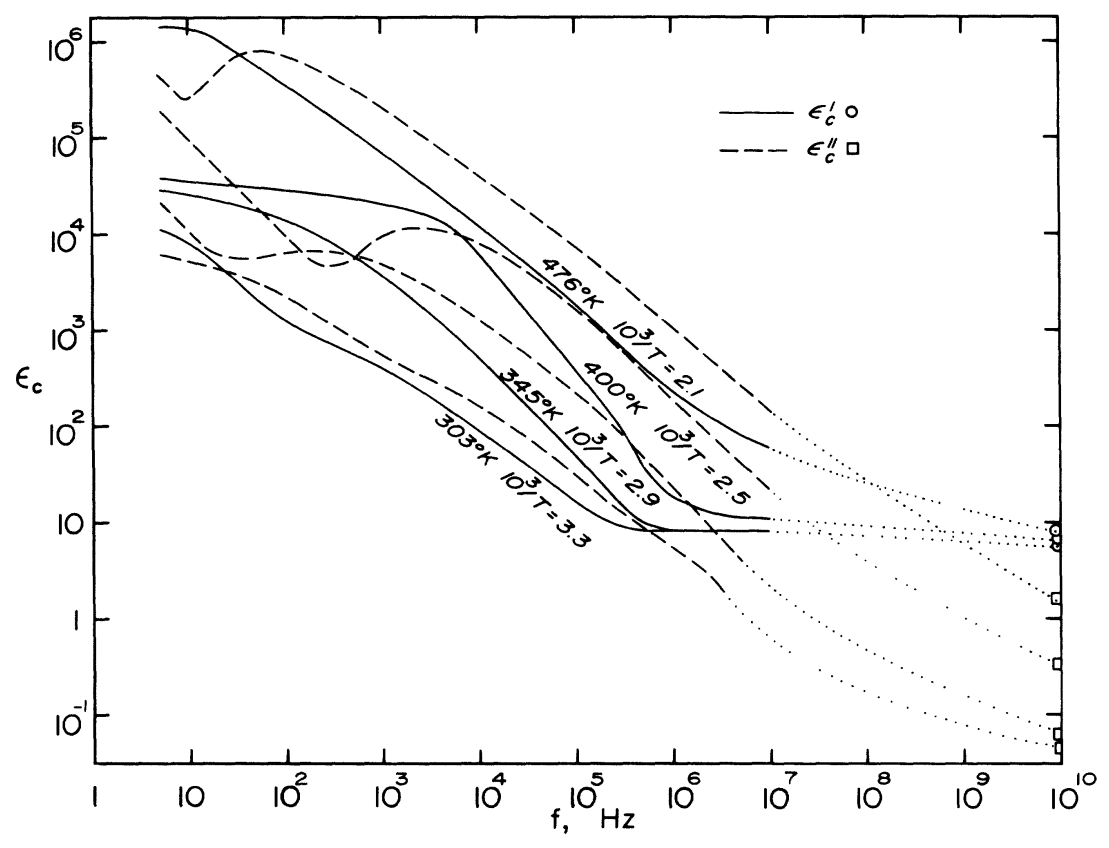

FIG. 12. Frequency dependence of dielectric constant for LiHzS No. 6, with points obtained for another LiHzS crystal at $9.330 \mathrm{GHz}$ shown also. Other experimental points are omitted for clarity. Dotted lines indicate frequencies for which no data were obtained.
-1 , as predicted by the random barrier model. At low frequency the diffusion to adjacent channels is expected to dominate the conductivity, and should cause a slope near -1 for $\epsilon^{\prime \prime}$. Such an increase in slope can be noted in some of the curves at about $10 \mathrm{~Hz}$. At higher frequencies, particularly above $100 \mathrm{kHz}$, the charge displacement per cycle is small and the barriers have less effect, causing the imaginary component to become larger than the real part as frequency increases, until the constant part $\epsilon_{h}^{\prime}$ takes over and prevents the real part from decreasing further. The portions of the $\epsilon^{\prime \prime}$ lines in Fig. 12, which are shown dotted in the frequency region between $10 \mathrm{MHz}$ and $9.33 \mathrm{GHz}$ in which no data were obtained, have a mean slope of less than -1 . This indicates that the conductivity of this crystal at 10 $\mathrm{MHz}$ has not reached its intrinsic value $\sigma_{i}$.

The $\epsilon^{\prime \prime}$ curves at high temperature show dips or flattening out somewhere in the $10 \mathrm{~Hz}$ to $1 \mathrm{kHz}$ region. This behavior is illustrated more dramatically in Fig. 15, in which it causes the dips near the right (low-frequency) ends of most of the lines. The calculated points also show this dip in the $476{ }^{\circ} \mathrm{K}$ graph. The dip occurs for that frequency interval having frequencies too high for interchannel diffusion to be important, and too low for the flow of charges back and forth between extrinsic barriers to contribute a large lossy component.

The rapid drops in $\delta$ at the left in Fig. 15 occur when $\epsilon^{\prime}$ has dropped to its high-frequency value $\epsilon_{h}^{\prime}$, while $\epsilon^{\prime \prime}$ continues to drop with increasing frequency.

The calculated points in Fig. 15 are for four different combinations of $E_{1}, E_{2}$, and $Q$. The "ref- erence" points are the squares, corresponding to $E_{1}=5, E_{2}=12$, and $Q=100$. Each of the other series of points is obtained by changing one of these parameters, keeping the other two fixed. The upright triangles show that the effect of increasing $E_{2}$ to 13 , and thereby decreasing the interchannel diffusion, is to decrease the lossy component considerably at high temperature and low frequency, while for other temperature-frequency combinations this increase has negligible effect. The diamonds show the effect of increasing $E_{1}$, which governs the carrier concentration, to 5.5 . This increase reduces both the real and imaginary components by about the same factor, keeping $\delta$ approximately constant. The amount of decrease is frequency independent, as one would expect. The inverted triangles illustrate the result of decreasing $Q$ to 50 , which corresponds to an eightfold increase in concentration of barrier-producing ions. The result at low frequency is about a tenfold reduction in both components of $\epsilon_{c}$, while at higher frequency it is only the imaginary component which is reduced. The outcome is a lower and shorter curve, giving an indication that LiHzS No. 5 has a higher barrier concentration than the other two crystals studied.

\section{DISCUSSION}

Ideally the parameters $E_{1}, E_{2}$, and $Q$ would first be determined by suitable measurements, after which the agreement between theory and experiment for the dielectric behavior could be found from plots such as Fig. 15. Unfortunately $Q$ is related to the unknown impurity ion concentration and $E_{2}$ is proportional to the interchannel diffusion activation energy 


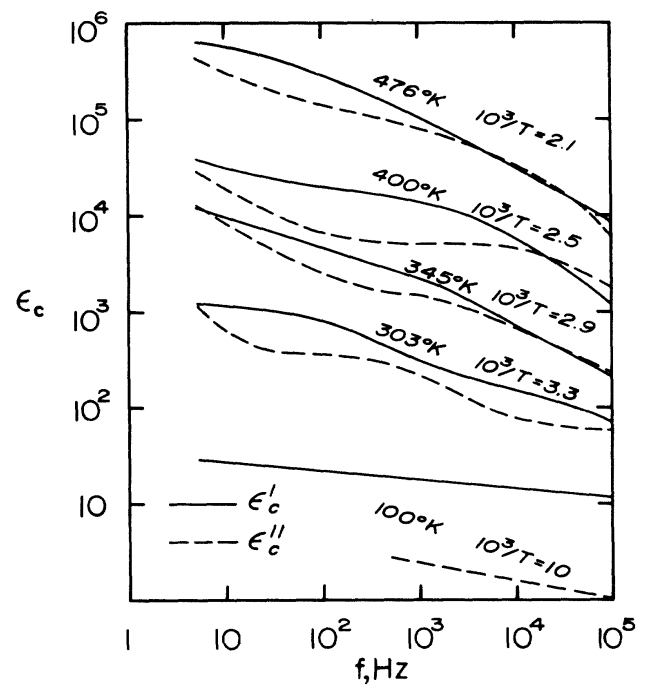

FIG. 13. Frequency dependence of dielectric constant for LiHzS No. 5 .

$u$, for which only limited and rather inconsistent information is available from the $a$ - and $b$-axis measurements.

With $E_{1}$ the situation is better, because it is proportional to the activation energy for the intrinsic $c$-axis conductivity $\sigma_{i}$ which occurs at frequencies high enough so that the extrinsic barriers have negligible effect. The values of 5 and 5.5 chosen for $E_{1}$ in Fig. 15 bracket the value obtained from the $\epsilon_{c}^{\prime \prime}$ activation energy at $9.330 \mathrm{GHz}$.

These values for $E_{1}$ fall quite close to those determined by NMR techniques for hindered rotation of $\mathrm{NH}_{2}$ groups about the $\mathrm{H}-\mathrm{N}-\mathrm{H}$ bisectrix in ordinary $^{10}$ and deuterated ${ }^{12}$ crystals. This close agreement seems significant at first glance, because one

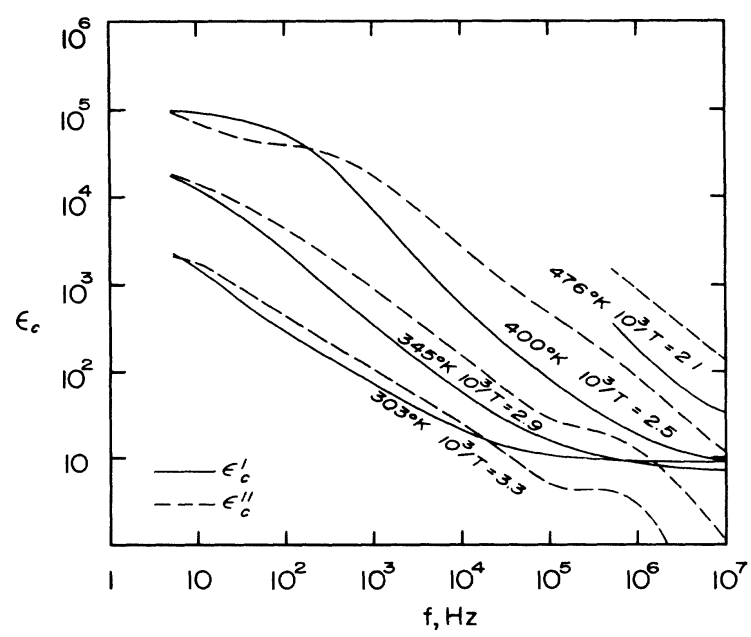

FIG. 14. Frequency dependence of dielectric constant for LiDzS No. 7. of the protons in the $\mathrm{NH}_{2}$ group forms part of the $\mathrm{N}-\mathrm{H} \cdot \cdots \mathrm{N}-\mathrm{H} \cdots$ chain along which protonic conduction is believed to occur. However, we believe that these activation energies are for two different processes, and only accidentally have nearly the same values. The deuteron $\mathrm{NMR}$ spectra from the $\mathrm{ND}_{2}$ groups merge between 280 and $330^{\circ} \mathrm{K}$ as a result of rapid hindered rotation averaging the electric field gradient for two D sites in each group. Similar averaging between a given $\mathrm{ND}_{2}$ group and its neighbors along the N-D..N-D... chain, sets in at $440^{\circ} \mathrm{K},{ }^{13}$ so hydrogen transfer from one $\mathrm{ND}_{2}$ to another along the $\mathrm{N}-\mathrm{D} \cdot \mathrm{N}$ bond occurs much less frequently than $\mathrm{ND}_{2}$ hindered rotation.

Because $E_{2}$ has effect only at lowest frequencies, the next parameter determined was $Q$. From Fig. 15 it appears that $Q$ near 100 fits the $\mathrm{LiDzS}$ and the LiHzS No. 6 data best, while $Q$ of about 50 gives

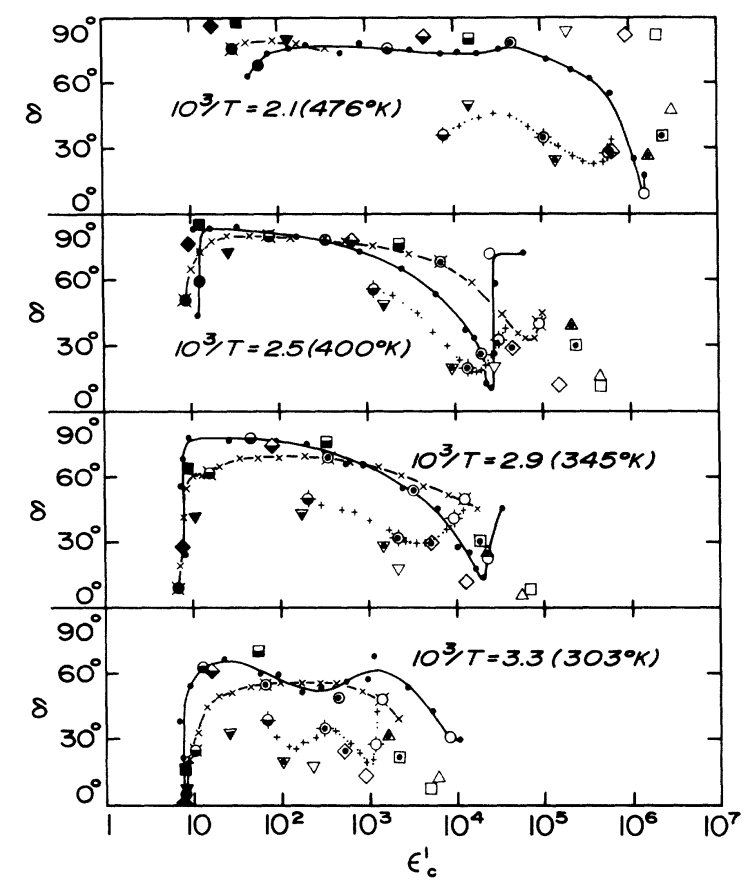

FIG. 15. Relation of loss angle $\delta\left(\tan \delta=\epsilon^{\prime \prime} / \epsilon^{\prime}\right)$ to real part of $c$-axis dielectric constant at four temperatures. The dotted lines and crosses are for LiHzS No. 5, solid lines and circles for LiHzS No. 6, and dashed lines and X's for LiDzS No. 7. Squares are points calculated by the random barrier height program for values 100,5 , and 12 for parameters $Q, E_{1}$, and $E_{2}$, respectively; triangles for $100,5,13$; diamonds for 100, 5.5, 12; and inverted triangles for $50,5,12$. Open symbols are for frequency of $10 \mathrm{~Hz}$, symbols with dots for $1 \mathrm{kHz}$, halffilled symbols for $100 \mathrm{kHz}$, and large filled symbols for $10 \mathrm{MHz}$. Crosses, X's, and small filled circles represent points at intermediate frequencies of $20 \mathrm{~Hz}, 50 \mathrm{~Hz}$, etc., up to $20 \mathrm{MHz}$. The triangles for $100 \mathrm{kHz}$ and 10 $\mathrm{MHz}$ are omitted for clarity, as they would nearly coincide with the corresponding squares. 
best agreement with the LiHzS No. 5 data points. If the extrinsic barriers result from uncompensated charges as assumed in deriving the barrier height distribution, the fractional concentration of such charges is about $10 / Q^{3}$ or $10^{-5}$ to $10^{-4}$. This is approximately $\frac{1}{100}$ of the measured ${ }^{17}$ impurity concentration. The long-range Coulomb fields from such charges would cause correlations of barrier locations for adjacent channels, and our neglect of such correlations would overemphasize the lossy part of the susceptibility. Alternatively the barriers may be caused by impurities with the correct valence (as $\mathrm{Na}^{+}$In $\mathrm{Li}^{+}$sites) or by locally compensated charges (as $\mathrm{Ca}^{++}$in $\mathrm{Li}^{+}$sites with nearly $\mathrm{H}^{+}$vacancies). In this case the assumption of no correlation for barrier locations becomes much better, but our barrier height distribution then must be considered as arbitrary. The fractional impurity concentration for this interpretation is much larger, and approaches $1 / Q$ in good agreement with the measured impurity concentration. The question of whether the barriers result from local distortions caused by impurities, or from Coulomb fields from a small fraction of impurities having uncompensated charges, could perhaps be resolved through experiments with doped crystals.

If the Coulomb barrier interpretation is correct, and if $E_{1}$ corresponds nearly to the activation energy for the carrier concentration $N$, with little or no activation energy for intrinsic $c$-axis mobility, then $N$ at all temperatures employed is less than the impurity ion concentration causing barriers and the original assumption that carriers do no screen impurity ions is justified. For barriers caused by local distortions this question of screening does not arise.

There remains the question of the value of $E_{2}$. Most of the experimental curves in Fig. 15 show an upturn at the right corresponding to the emergence of interchannel diffusion as the governing loss mechanism at low frequency. To obtain similar upturns for the calculated curves, it is necessary to reduce $E_{2}$ considerably with decreasing temperature. Our activation energies for dc conductivity along $a$ and $b$ in LiDzS show such decrease with decreasing temperature, and $\mathrm{LiHzS}$ also has conductivity in the $a$ and $b$ directions which cannot be described by a single activation energy. ${ }^{2}$ A possible explanation is that defects or impurities cause a small number of permanent low interchannel barriers. At low temperature these low barriers govern interchannel diffusion, while at higher temperature the flow across the large number of ordinary barriers of height $v$, or perhaps across lower barriers thermally induced by a reversible process, exceeds that across the few low barriers. The values of $E_{2}$ giving the observed upturn at the right vary from 13 at $476{ }^{\circ} \mathrm{K}$ to 9 at $345{ }^{\circ} \mathrm{K}$ for the undeuterated crys- tals, or 1.12 to $0.78 \mathrm{eV}$. This decrease in $E_{2}$ is probably related to the decreases in activation energies for dc conductivity along $a$ and $b$ in $\mathrm{LiHzS}$ and LiDzS described in Sec. IV.

The random barrier model for one set of parameters and one temperature is compared in Fig. 4 with experiment and with the uniform barrier case. The parameters $Q_{u}$ and $u$ for this case obey two conditions which give some physical correspondence to the random barrier model. Roughly speaking, we retain only those random barriers high enough so that diffusion around them via interchannel jumps becomes significantly at low frequency. These barriers then are given equal separation $Q_{u} c$ and equal height $u$. Specifically, one condition is equality of the last two terms in Eq. (15) corresponding, respectively, to jumps across and diffusion around the extrinsic barriers. The other condition is that $Q_{u} c$ must be the average separation of barriers of height $u$ or greater in the random barrier model, where from Sec. II, $u=u_{s} w_{u}^{-1 / 2}$ and $Q_{u}=Q w_{u}{ }^{-1 / 2}$. Only a few barriers have the necessary height, so $Q_{u}$ is quite large. The agreement with experiment is evidently better for random barriers than for uniform barriers.

A brief comparison of LiHzS with related materials follows. Closest related chemically are other hydrogen-bonded crystals. Those previously studied show protonic semiconductivity with the activation energies and room-temperature conductivities in the same range as for LiHzS. Most are nearly isotropic conductors, but others such as borax ${ }^{23}$ have different mobilities along the three principal axes of the conductivity tensor. The $c$-axis conductivity of LiHzS is similar to that of ice, ${ }^{24}$ and presumably would be much larger in the absence of extrinsic barriers. LiHzS has one-dimensional $\mathrm{N}-\mathrm{H} \cdots \mathrm{N}-\mathrm{H} \cdots$ chains, while ice has a three-dimensional $\mathrm{O}-\mathrm{H} \cdots \mathrm{O}-\mathrm{H}$... network.

The ac dielectric properties of ice and other crystals such as potassium dihydrogen phosphate ${ }^{25}$ (KDP) which have fairly large dielectric susceptibility generally are caused by dipole reorientation (caused by $\mathrm{H}_{2} \mathrm{O}$ rotation and hydrogen intrabond motion, respectively, in ice and KDP), and can be approximately described with a single relaxation time. In LiHzS the $\mathrm{NH}_{2}$ and $\mathrm{NH}_{3}{ }^{+}$rotations are to electrically equivalent positions, and the only local motion which is expected to affect the dielectric behavior somewhat is reversal of a small fraction of the $\mathrm{N}-\mathrm{H}$ dipoles in the $\mathrm{N}-\mathrm{H} \cdots \mathrm{N}-\mathrm{H} \cdots$ chains. ${ }^{14}$ The major contribution to the LiHzS $c$-axis dielectric susceptibility is from conduction along partially blocked channels, and it has a very broad distribution of relaxation times. ${ }^{19}$

In comparing LiHzS with ferroelectrics, the latter often have very large dielectric susceptibilities near the Curie point, but LiHzS has a large and 
lossy susceptibility over a wide temperature range. In high fields it exhibits apparent hysteres is loops which result from dielectric saturation in conjunction with dielectric loss, both phenomena being aspects of the partially blocked conduction.

The dielectric effect of blocked conducting channels was implicitly noted long ago by Sillars, ${ }^{26}$ who pointed out that metal needles in an insulator have a much greater effect than the same volume of metal distributed as small spheres. Effects similar to those noted in LiHzS should occur in any "onedimensional conductor." To our knowledge, the only other report of a "one-dimensional conductor" is a recent letter ${ }^{27}$ on electronic dc conduction in the square planar complex $\mathrm{K}_{2} \mathrm{Pt}\left(\mathrm{CN}_{4}\right) \mathrm{BR}_{0.30} \cdot 2.3 \mathrm{H}_{2} \mathrm{O}$, in which ac behavior and defects which might block the conduction paths were not considered.

Behavior phenomenologocally similar to that in $\mathrm{LiHzS}$ has been reported for certain polymers containing long molecules. ${ }^{28}$ The carriers (electrons or holes) can travel easily along the molecules by means of extended orbitals, but must jump across or tunnel through barriers which exist between molecules. Different molecules are oriented in different directions, so the behavior is isotropic. The dielectric constant is large and lossy, increases exponentially with temperature and pressure, and has approximately $f^{-0.8}$ frequency dependence.

\section{CONCLUSIONS}

The LiHzS crystal structure has channels which allow easy flow of current along only one axis. In such a "one-dimensional conductor," local defects have two pronounced effects on carrier mobility which are nearly absent in isotropic conductors. As one would expect, both effects tend to make the conduction more isotropic.

One effect, caused by extrinsic barriers within channels, is a decrease in conductivity with decreasing frequency accompanied by a phase shift which gives a very large real as well as imaginary component of dielectric susceptibility. Our model which postulates a particular barrier height distribution is in good qualitative agreement with our experimental observations of this effect.

The other effect, resulting from anomalously low barriers between channels near certain defects, is an increase in interchannel diffusion. Experimentally two manifestations of this effect have been tentatively identified in the conductivity perpendicular to the channels. At low temperature this conductivity has low activation energy and anomalously small $\sigma_{0}$ because of a fixed number of low barriers, while at high temperature the activation energy is large and $\sigma_{0}$ is anomalously large because of thermally induced low barriers. Effects of this activation energy change appear also in the low-frequency dielectric susceptibility along the channels.
We have shown that the unusual dielectric properties of LiHzS result from its highly anisotropic nearly one-dimensional conductivity which is extremely sensitive to defects. The large-signal dielectric phenomena which include apparent hysteresis loops are now being studied. Crystals for further dielectric studies are being grown with varying concentrations of impurities and defects to permit a detailed study of the mechanisms by which the defects determine the dielectric properties.

\section{ACKNOWLEDGMENTS}

We wish to thank D. W. Berg, Dr. R. R. Knispel, and R. S. Parker for assistance in obtaining data. Berg also gave valuable help in writing the computer program. We are indebted to Dr. H. Arend of the Swiss Federal Technical University (ETH) for the analysis of the decomposition rate, and to Dr. R. Bärtschi of the Swiss Federal Institute for Reactor Research for determining the degree of deuteration of the crystals.

\section{APPENDIX}

In addition to the case of partially blocking extrinsic barriers presented in Sec. II, three other exactly soluble cases have been examined which are presented here. These have completely blocking barriers at regular intervals in a given channel. The first has separation $4 q$ between blocking barriers, with intervening barriers of height $u$. The second and third have separation $6 q$ between blocking barriers, with two intervening barriers between each pair of blocking barriers. The intervening barriers are of equal height $u$ for the second case, and of unequal heights $u_{1}$ and $u_{2}$ for the third.

The blocking barriers provide convenient boundary conditions which allow calculation of the susceptibility from the behavior of the region between only one pair of blocking barriers. These conditions are that $J(-q)=0$ at the left end of the first segment and $J(q)=0$ at the right end of the last segment, using Eq. (9) for $J$. These two conditions, together with the conditions in Eqs. (10) and (11) for each finite barrier, are sufficient to determine the $n_{s m}$ and $n_{c m}$, from which $\chi_{c j}$ is found using Eq. (12).

The susceptibility for alternate blocking and partially blocking barriers is

$$
\chi_{c j}=\frac{\sigma_{i}}{i \omega}\left(1-\frac{\tanh k q}{k q} \frac{1+\frac{1}{2}(3 A-B+1) U}{1+(A+B) U}\right) .
$$

The susceptibility for blocking barriers separated by two intervening partial barriers of equal height $u$ is

$$
\chi_{c j}=\frac{\sigma_{i}}{i \omega}\left(1-\frac{\tanh k q}{k q} \frac{1+\frac{2}{3}(A-1) U}{1+2 B U}\right) .
$$

The more general case for two intervening barriers of heights $u_{1}$ and $u_{2}$ has 
$\chi_{c j}=\frac{\sigma_{i}}{i \omega}\left(1-\frac{\tanh k q}{k q}\right.$

$$
\left.\times \frac{1+\frac{1}{3}(4 A-1)\left(U_{1}+U_{2}\right)+\frac{4}{3} A(A-1) U_{1} U_{2}}{1+(A+B)\left(U_{1}+U_{2}\right)+4 A B U_{1} U_{2}}\right) .
$$

In these expressions

$$
\begin{aligned}
& A=(2 \cosh 2 k q+1) / k c \sin 2 k q, \\
& B=(2 \cosh 2 k q-1) / k c \sinh 2 k q, \quad U_{m}=e^{-\beta u_{m}} .
\end{aligned}
$$

For $k q \gg 1$, these parameters have approximate values $A \simeq B \simeq 2 / k c$ and $\tanh k q \ll k q$, so $\chi_{c j} \simeq \sigma_{i} / i \omega$ and the susceptibility in each case is controlled by the intrinsic conductivity, as was found also for equal barrier heights for $k q \gg 1$. For $k q \ll 1$, the approximate values become $A \simeq 3 B \simeq 3 / 2 k^{2} q c \gg 1$, so $\chi_{c j} \ll \sigma_{i} / i \omega$ and it becomes desirable as well as practical to develop approximate expressions for $\chi_{c j}$, using also the assumption that $q \gg c$. These low-frequency susceptibilities are

$$
\chi_{c j}=\frac{Q^{2} \sigma_{i}}{3 \nu} \frac{\gamma+i \omega}{i \omega} \frac{4 \delta+\gamma+i \omega}{\delta+\gamma+i \omega},
$$

for the barrier factor $\left(U_{m}\right)$ sequence $0, U, 0, U, \cdots$,

$$
\chi_{c j}=\frac{Q^{2} \sigma_{i}}{3 \nu} \frac{\gamma+i \omega}{i \omega} \frac{\frac{9}{2} \delta+\gamma+i \omega}{\frac{1}{2} \delta+\gamma+i \omega},
$$

for the sequence $0, U, U, 0, U, U, \cdots$, and

$$
\begin{aligned}
\chi_{c j}= & \frac{Q^{2} \sigma_{i}}{3 \nu} \frac{\gamma+i \omega}{i \omega} \\
& \times \frac{\left(\frac{9}{4} \delta_{1}+\gamma+i \omega\right)\left(\frac{9}{4} \delta_{2}+\gamma+i \omega\right)-\frac{1}{4}(\gamma+i \omega)^{2}}{\left(\frac{3}{4} \delta_{1}+\gamma+i \omega\right)\left(\frac{3}{4} \delta_{2}+\gamma+i \omega\right)-\frac{1}{4}(\gamma+i \omega)^{2}},
\end{aligned}
$$

for the sequence $0, U_{1}, U_{2}, 0, U_{1}, \cdots$. In these expressions $Q=2 q / c, \nu=\nu_{0} e^{-\beta u_{0}}, \delta_{m}=\nu U_{m} / Q$, and $\gamma=4 \nu_{0} e^{-\beta v}$.

A feature common to all four exact models is the dominance of the imaginary component $\chi_{c j}^{\prime \prime}$ at the lowest and highest frequencies. At high frequencies the intrinsic conductivity dominates, giving $\chi_{c j}$ $\simeq \sigma_{i} / i \omega$. At low frequency the dominant factor is the conductivity which exists despite the blocking barriers, by virtue of the carrier diffusion to adjacent channels. At intermediate frequencies, as the variability in barrier heights increases in going from the first to the last model, the real and imaginary components become more nearly equal and tend more and more toward the observed $\omega^{-x}$ frequency dependence, where $0<x<1$.
${ }^{\dagger}$ Work supported by National Science Foundation.

*On sabbatical leave during 1971 at Laboratorium für Festkörperphysik, Eidgenössische Technische Hochschule, Zurich, Switzerland.

${ }^{1}$ R. Pepinsky, K. Vedam, Y. Okaya, and S. Hoshino, Phys. Rev. 111, 1467 (1958).

${ }^{2}$ J. Vanderkooy, J. D. Cuthbert, and H. E. Petch. Can. J. Phys. 42, 1871 (1964).

${ }^{3}$ N. Niizeki and H. Koizumi, J. Phys. Soc. Japan $\underline{19}$, 132 (1964).

${ }^{4}$ I. D. Brown, Acta Cryst. 17, 654 (1964).

${ }^{5} \mathrm{~J}$. H. Van den Hende and H. Boutin, Acta Cryst. 17 , 660 (1964).

${ }^{6}$ V. M. Padmanabhan and R. Balasubramanian, Acta Cryst. 22, 532 (1967).

${ }^{7} \mathrm{~F}$. Ross (private communication).

${ }^{8}$ J. D. Cuthbert and H. E. Petch, Can. J. Phys. 41 , 1629 (1963).

${ }^{9}$ W. D. MacClement, M. Pintar, and H. E. Petch, Can. J. Phys. 45, 3257 (1967).

${ }^{10}$ R. R. Knispel and H. E. Petch, Can. J. Phys. $\underline{49}$, 870 (1971).

${ }^{11} \mathrm{~F}$. L. Howell and V. H. Schmidt, J. Chem. Phys. 51, 1983 (1969).

${ }^{12}$ V. H. Schmidt and F. L. Howell, J. Phys. Soc. Japan Suppl. 28, 106 (1970).

${ }^{13}$ R. S. Parker, Ph. D. thesis (Montana State Univer- sity, 1971) (unpublished).

${ }^{14}$ V. H. Schmidt, J. Math. Phys. 12, 992 (1971).

${ }^{15} \mathrm{R}$. Bärtschi (private communication).

${ }^{16} \mathrm{~J}$. Muheim (private communication).

${ }^{17}$ F. L. Howell, Ph. D. thesis (Montana State University, 1969) (unpublished).

${ }^{18} \mathrm{~A}$. Van Hippel, Dielectric Materials and Applications (Wiley, New York, 1954), Chap. II.

${ }^{19}$ P. Wan, F. L. Howell, V. H. Schmidt, and J. E. Drumheller, Bull. Am. Phys. Soc. 15, 606 (1970).

${ }^{20}$ For a recent extensive review of measurement techniques see H. E. Bussey, Proc. IEEE 55, 1046 (1967).

${ }^{21} \mathrm{~S}$. Devanarayanan and K. R. K. Easwaran, Proc. Indian Acad. Sci. 64A, 173 (1966).

${ }^{22} \mathrm{H}$. A rend (private communication).

${ }^{23}$ S. Maricic, V. Pravdic, and Z. Veksli, J. Phys. Chem. Solids 23, 1651 (1962); E. W. Giesekke and L. Glasser, J. Phys. Chem. $\underline{71}, 2573$ (1967).

${ }^{24} \mathrm{~N}$. H. Fletcher, The Chemical Physics of Ice (Cambridge U. P., London, 1970).

${ }^{25}$ V. H. Schmidt, Phys. Rev. 164, 749 (1967).

${ }^{26}$ R. W. Sillars, J. Inst. Elec. Engrs. (London) 80 , 378 (1937).

${ }^{27}$ M. J. Minot and J. H. Perlstein, Phys. Rev. Letters $\underline{26}, 371$ (1971).

${ }^{28}$ R. Rosen and H. A. Phl., J. Polymer Sci. 4, 1135 (1966). 